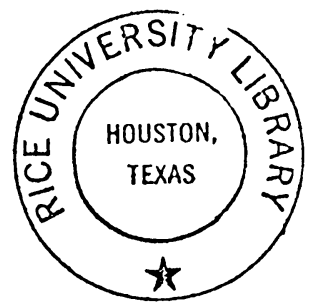

\title{
RICE UNIVERSTTY
}

THE EFFECTS OF UPSTREAM MASS INJECTION

ON DOWNISTREAN HEAT TRAINSFER

by

W. Robert Wolfram, Jr.

A THESIS SUBIIITTED

IN PIRTIAL TTLFILIIENT OF THE

REOUIREMEITS FOR THE DHGREE OF

Master of Science

Thesis Director's signature:

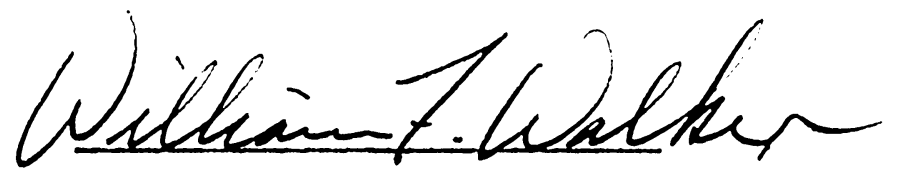

Houston, Texas

Nay, 1969 
ABSTRACT

THE EFFECTS OF UPSTREAII MASS INJECTION

ON DOWNSTREAIY HEAT TRAINSFER

by

W. Robert : Volfram, Jr.

This study was performed in order to determine the effects of upstream mass injection on dormstream heat transfer in a laminar nonreacting boundary layer. The study differs from numerous previous investigations in that no similarity assumptions are made. A numerical technique knom as the method of integral-matrix analysis is used. This approach is a recent outgrowth of the method of integral relations. The complete coupled set of non-reacting laminar boundary layer equations with discontinuous mass injection was solved for this problem using the integral-matrix technique. The effects of mass injection on heat transfer to both sharp and blunt-nosed isothermal flat plates were studied for a Nach 2 freestream. The amount of injection and the length of the injected region were varied for each body. Heat transfer rates were found to decrease markedly in the injected region. A sharp rise in heat transfer was found immediately downstream of the region of injection followed by an asymptotic approach to the heat transfer rates calculated for the case of no injection. An insulating effect was found to persist for a considerable distance domstream of the injection region. The distance required for this insulating effect to die out was found to depend on the length of the injection region as well as the rate of injection. 


\section{ACKNOIDEDGMENT}

I wish to express my thanks to Dr. William F. Walker, my thesis advisor, for all his help and inspiration in defining and solving the problem. I am also indebted to the National Aeronautics and Space Administration for both their generous financial support and for use of their computing facilities. Finally I wish to thank my wife Melinda for her patience in typing the manuscript. 
TABLE OF CONTENTS

Page no.

NOMENCLATURE

$i$

I. INTRODUCTION

1

II. ANALYSIS

5

III. RESULTS AND DISCUSSION 19

IV. CONCLUSIOHS 23

V. BIBLIOGRAPHY 24

VI. APPEINDIX 


\section{NONENCLATURE}

$A_{1}, A_{2}, A_{3}, A_{4}=$ coefficients defined by equations (40)

$B_{1}, B_{2}, B_{3}, B_{4}=$ coefficients defined by equations (44)

$C_{1}, C_{2}, C_{3}, C_{4}=$ coefficients defined by equations (45)

$c=$ product of density and viscosity normalized by their freestream values

$C_{p}=$ heat capacity

$d_{0}, d_{1}, d_{2}=$ coefficients defined by finite difference relations ( 34 )

$D_{12}=$ binary diffusion coefficient

$f=$ stream function

$g$ = the velocity ratio specified at a control node

$h=$ static enthalpy

$\mathrm{H}_{t}=$ total enthalpy defined by $\mathrm{H}_{t}=\mathrm{h}+\mathrm{u}^{2} / 2$

$k=$ thermal conductivity

$K_{i}=$ mass fraction of the $i$ th species

$m=$ molecular weight of the mixture

$m_{i}=$ molecular weight of the $i$ th species

$\dot{n}_{\mathrm{W}}=\rho_{\mathrm{W}} \mathrm{V}_{\mathrm{W}}=$ mass rate of injection.

$N=$ number of nodal points across the boundary layer

$\mathrm{N}_{\mathrm{pr}}=$ Prandtl number $=\mathrm{C}_{\mathrm{p}} \mu / \mathrm{k}$

$\mathrm{N}_{\text {sc }}=$ Schmidt number $=\mu / \rho \mathrm{D}_{12}$

$P=$ dummy variable

$\mathrm{p}=$ static pressure

$\dot{q}_{n}=$ local heat transfer rate for the zero injection case

$\dot{q}_{s t}=$ stagnation point heat transfer rate 


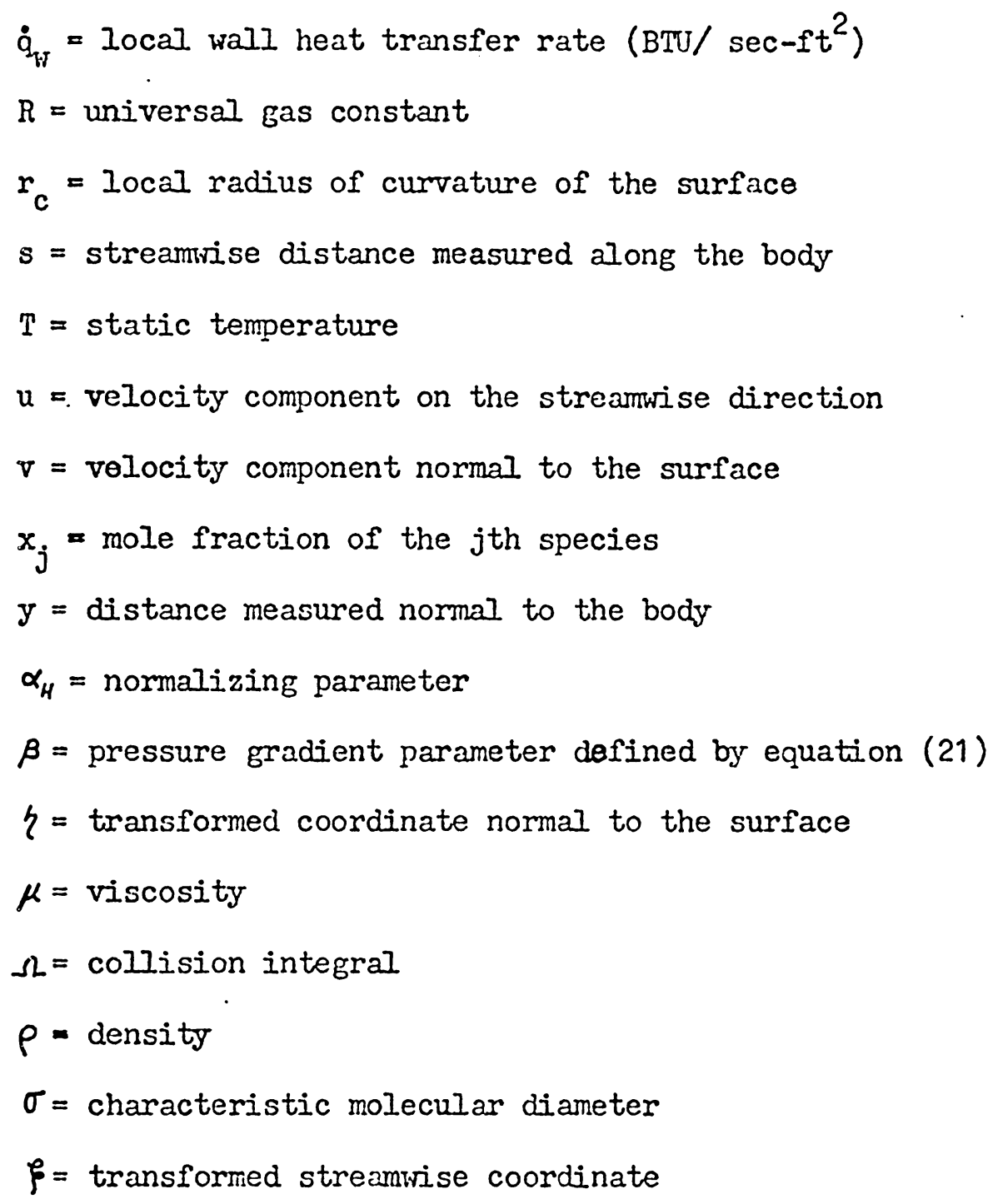




\section{INTRODUCTION}

The following study was undertaken in order to determine the effects of upstream mass injection on the downstream heat transfer properties of a supersonic laminar boundary layer. High velocity lifting entry vehicles require considerable ablation or mass injection cooling in the stagnation region in order to overcome the large heat transfer rates produced by the severe thermal environment during entry. This injected mass remains in the boundary layer as the flow accelerates around the body. However, additional mass injection or even thermal sampling may be taking place on the afterbody of the vehicle. Therefore, it is desirable to determine to what extent the insulating properties of the upstream injection persist domstream in the boundary layer flow.

The study has further application in such problems as injection cooling near the leading edge of high-speed airfoils. In this case it is desirable to keep the actual region of injection small due to structural considerations. Downstream persistence of an insulating effect from injection would then be highly beneficial.

The Iaminar boundary layer with mass injection has been the subject of numerous investigations in the past. Most of these studies have treated flow in the region of injection and flow over an impermeable surface domstream from the injection region as tivo separate problems. The solutions obtained required some type of matching of solutions at the interface between the injection region and the downstream region. 
Solutions for the injected region have usually been of the similarity type. Such a solution was obtained by Iow $(1)$, who performed a classical similarity analysis for the compressible laminar boundary layer with continuous fluid injection. This solution has often been cited as the "exact" solution and has usually formed the starting point for studies of flow domstream from an injection region. However, similarity requires the rather restrictive assumption that the rate of coolant injection, $\dot{\mathrm{m}}_{\mathrm{W}}$, be proportional to some power of the distance from the leading edge. In this case the power was $\left(\frac{1}{2}\right)$. Otherwise, boundary conditions cannot be transformed properly. Lee and sundell(2) showed experimentally that $i_{W}$ is nearly constant with distance for the case of ablation of Teflon from an Apollo-shaped body. This result casts serious doubts at least on the validity of the similarity assumptions.

More recently, Smith \& Clutter ${ }^{(3)}$ developed a finite difference solution to the laminar boundary layer problem. Their numerical procedure was reported to contain the capability of handling arbitrary distributions of mass injection. However, their investigation was directed more toward other aspects of the boundary layer problem and no applications to mass transfer cooling were presented.

The dormstream region has been studied mainly by use of the KarmanPohlhausen integral approach. Rubesin and Inouye (4) used this method with a seventh-degree polynomial approximation to the velocity and temperature profiles. They matched their profiles to Low's solution at the interface by assuming shear stress and boundary layer thickness 
to be continuous at the junction. Libby and Pallone ${ }^{(5)}$ used sixthdegree polynomials but introduced additional parameters to insure continuity of mass, momentum, and energy at the interface. The profiles were allowed to change discontinuously at the interface but the parameters were not made functions of distance. The solution is, therefore, valid only for a short distance domstream from the injected region. Howe ${ }^{(6)}$ used a finite difference scheme in the domstream region but again matched to Low's solution at the junction. In comparing his results with those of Rubesin and Inouye and Libby and Pallone, he found significant differences among the solutions. All three studied the rise in wall temperature along an insulated plate. Howe's results fell in between the optimistic results of Libby and Pallone and the more conservative findings of Rubesin and Inouye.

Chung ${ }^{(7)}$ and Cresci $^{(8)}$ have followed similar procedures for axisymmetric bodies. Chung assumed seventh-degree polynomial profiles while Cresci used exponential profiles.

Pallone ${ }^{(9)}$ has also studied vall temperature rise along an insulated plate, using Low's solution in the porous region. His analysis is significant in that it more nearly follows the method of integral relations used in the present analysis. Instead of integrating across the whole boundary layer, as is done in the Karman-Pohlhausen approach, he subdivided the boundary layer into is separate strips. Integration of the governing equations was then performed across each strip, using a polynomial to represent the integrand over each individual strip. This resulted in far greater accuracy than is possible vith the more 
gross momentum-integral method. The resulting ordinary differential equations were integrated numerically and the results compared well with Howe's finite difference results.

The present study differs from the previous ones in several respects. First of all, many of the restrictive assumptions of previous investigators were relaxed. No similarity was assumed for the region of injection, thus allowing arbitrary distributions of mass injection. Hass injection was taken to be constant over the first portion of a planar body, followed by a region of zero injection. The complete nonsimilar, compressible laminar boundiry layer equations were solved by use of the integralmatrix technique ${ }^{(10)}$. This method is a recent outgrowth of the method of integral relations ${ }^{(11)}$. Both the injection and dormstream regions were solved together as one problem.

The effects of mass injection on heat transfer to an isothermal plate were studied. Both sharp and blunt-nosed configurations vere considered and the results compared. 


\section{ANALYSIS}

The equations that govern the physical system under consideration are the tro-dimensional, steady-state, non-reacting, laminar boundary layer equations.

Continuity: $\frac{\partial}{\partial s}(\rho u)+\frac{\partial}{\partial y}(\rho v)=0$

Streamise lomentum: $\rho u \frac{\partial u}{\partial s}+\rho v \frac{\partial u}{\partial y}+\frac{\partial P}{\partial s}=\frac{\partial}{\partial y}\left(\mu \frac{\partial u}{\partial y}\right)$

Normal Nomentum: $\quad\left(\frac{\partial P}{\partial y}\right)_{S}=\frac{\rho u^{2}}{r_{c}}$

Species Conservation: $\rho u \frac{\partial K_{i}}{\partial S}+\rho v \frac{\partial K_{i}}{\partial y}=\frac{\partial}{\partial y}\left(\rho D_{12} \frac{\partial K_{i}}{\partial y}\right)$

Energy: $\rho \mu \frac{\partial H_{t}}{\partial S}+\rho v \frac{\partial H_{t}}{\partial y}=\frac{\partial}{\partial y}\left(\mu \frac{\partial\left(u^{2} / 2\right)}{\partial y}+k \frac{\partial T}{\partial y}\right)$

$$
+\frac{\partial}{\partial y}\left(\rho D_{12} \frac{\partial h}{\partial y}\right)
$$

where $K_{i}$ is the mass fraction of the $i$ th species, $D_{12}$ is the binary diffusion coefficient, $\mu$ is the mixture viscosity, $k$ is the thermal conductivity, and $\mathrm{H}_{t}$ is the total enthalpy.

It should be noted at this point that thermal diffusion is neglected and no internal generation of species is assumed. Also, as the radius of curvature, $r_{c}$, will alvays be large cormared with the boundary layer thickness, the term $\left(\frac{\partial P}{\partial y}\right)_{s}$ (equation (3)) becomes approximately equal to zero. Therefore, it may be concluded that there is no normal pressure gradient, and the pressure becomes a function of streamiise distance only. In all cases of interest to the present study the system was assumed to be a binary mixture of $78.8 \% \mathrm{~N}_{2}$ and $21.2 \% \mathrm{O}_{2}$ by volume. 
The boundary conditions are as follows:

1. $u_{w}=0$

2. $\dot{m}_{W}=\rho_{W} v_{W}$, a given function of $s$

3. $T_{W}=a$ given function of $s$

4. $K_{i w}=$ given

5. Edge conditions are specified functions of $\mathbf{s}$

$$
u_{\infty}(s), p_{\infty}(s), T_{\infty}(s), K_{i \infty}(s)
$$

where subscript "w" denoted the wall (i.e. $y=0$ ).

The transport properties are treated in accordance with kinetic theory (12) with slight modifications reported in reference (10). Equal diffusion coefficients are assumed vith $\mathrm{D}_{12}$ given by the following relation:

$$
D_{12}=2.628 \times 10^{-3} \frac{T\left(T / m_{\text {ref }}\right)^{1 / 2}}{p \sigma_{\text {ref }}^{2} \Omega_{\text {ref }}^{(1,1)}}\left(\mathrm{cm}^{2} / \mathrm{sec}\right)
$$

where $\mathrm{T}$ is the temperature in degrees Kelvin, $\mathrm{p}$ is the pressure in atm., $M_{\text {ref }}$ is the molecular weight of the reference species, $\sigma_{\text {ref }}$ is the characteristic diameter of a molecule of the reference species (angstrom units), and $\Omega_{\text {ref }}^{(1,1)}$ is the collision integral for the reference species, either $\mathrm{O}_{2}$ or $\mathrm{N}_{2}$ since the binary diffusion coefficients are assumed equal for both species.

The viscosity is obtained from the Sutherland-idussiljera

approximation:

$$
\mu=\sum_{i=1}^{2} \mu_{i} D_{i}
$$

where

$$
\mu_{i}=\left(\frac{5}{6} \frac{\Omega_{i}^{(1,1)}}{\Omega_{i}^{(2,2)}}\right) \rho D_{12}
$$

with

$$
D_{i}^{-1}=1.0+1.385 \frac{R T \mu_{i}}{P x_{i} m_{i}} \frac{x_{j}}{D_{12}}
$$

where $x_{j}=$ mole fraction of $j$ th species $(i \neq j)$. 
The thermal conductivity, $k$, is treated as the sum of the monotonic thermal conductivity and a contribution from the internal degrees of freedom:

$$
k=k^{\text {mono }}+k^{\text {int }}
$$

where $k^{\text {mono }}=\sum_{i=1}^{2} k_{i}^{\text {mono }} D_{i}^{*}$

with $k_{i}^{\text {mono }}=\left(15 R / 4 m_{i}\right) \mu_{i}$

and $\quad D_{i}^{x^{-1}}=1.0+1.065 \times 1.385 \frac{R T \mu_{i}}{P x_{i} m_{i}} \frac{x_{i}}{D_{12}}$

$$
(i \neq j)
$$

The contribution from the internal degrees of freedom is given by:

$$
k^{\text {int }}=\sum_{i=1}^{2} x_{i}\left(k_{i}-k_{i}^{\text {mono }}\right)
$$

where

$$
k_{i}-k_{i}^{\text {mono }}=P D_{12} \frac{m_{i}}{m}\left(C_{P_{i}}-\frac{5}{2} \frac{R}{m_{i}}\right)
$$

The temperature dependencies of $c_{p}, h$, and entropy are obtained from curve fits of JANAF experimental data (13). 
TRANSFORMATI ONS: A modified Levy-Lees similarity transform is used with non-similar terms being retained in the equations. The transformed variables $\ell$ and $\xi$ are given by ${ }^{(10)}$ :

$$
\begin{aligned}
& \xi=\frac{u_{\infty}(\xi)}{\alpha_{H}(\xi) \sqrt{2 \xi}} \int_{0}^{y} P(y) d y \\
& \xi=\int_{0}^{s} u(s) \rho_{\infty}(s) \mu_{\infty}(s) d s
\end{aligned}
$$

The quantity $\alpha_{H}(\xi)$ is a stretching parameter determined during the numerical iteration. It is used to keep the boundary layer thickness relatively uniform in the transformed plane. This is mainly a numerical convenience in that a uniform placement of nodal points across the boundary layer can be set in advance for all problems. The parameter

$\alpha_{H}(\xi)$ is determined by specifying the velocity ratio at a certain nodal point in the boundary layer.

$$
\left.f^{\prime}\right|_{\eta_{c}}=\left.g f^{\prime}\right|_{\eta_{\infty}}
$$

where

$$
f^{\prime}=\alpha_{H} u / u_{\infty}
$$

A good choice has been $g=0.80$ at the 5 th of seven nodes.

A stream function is also defined and is given by:

$$
f-f_{\omega}=\alpha_{H} \int_{0}^{\eta} \frac{u}{u_{\infty}} d \xi
$$

Applying these transformations to equations (2), (4) and (5) yields the results:

$$
\text { Momentum: } \begin{aligned}
& f f^{\prime \prime}+\left[\frac{c f^{\prime \prime}}{\alpha_{H}}\right]^{\prime}+\beta\left(\alpha_{H}^{2} \frac{\rho_{\infty}}{\rho}-f^{\prime 2}\right) \\
& =2\left(f^{\prime} \frac{\partial f^{\prime}}{\partial \ln \rho}-f^{\prime \prime} \frac{\partial f}{\partial \ln \xi}-f^{\prime 2} \frac{d \ln \alpha_{H}}{d \ln \xi}\right)
\end{aligned}
$$


where ' denotes $\frac{\partial}{\partial \eta}$

and where $c=\rho \mu / \rho_{\infty} \mu_{\infty}$

and

$$
\beta=2 \frac{d \ln u_{\infty}}{d \ln \xi}
$$

The quantity $\beta$ therefore is a pressure gradient parameter.

Species: $f K_{i}^{\prime}+c \frac{1}{N_{s c}} K_{i}^{\prime}=2 \xi\left[f^{\prime} \frac{\partial K_{i}}{\partial \xi}-K_{i}^{\prime} \frac{\partial f}{\partial \rho}\right]$

Energy: $f H_{t}^{\prime}+\frac{c}{\alpha_{H}}\left[\frac{f f^{\prime \prime}}{\alpha_{H}^{2}} u_{\infty}^{2}+\frac{C_{P}}{N_{P R}} T^{\prime}+\frac{h^{\prime}}{N_{S C}}\right]^{\prime}$

$$
=2 \xi\left[f^{\prime} \frac{\partial H_{t}}{\partial \xi}-H_{t}^{\prime} \frac{\partial f}{\partial \xi}\right]
$$

The boundary conditions are similarly transformed to give:

$$
\begin{aligned}
& f_{w}=-(2 \xi)^{-1 / 2} \int_{0}^{\xi} \frac{\rho_{w} v_{w} d \xi}{\rho_{\infty} u_{\infty} \mu_{\infty}} \\
& f_{w}^{\prime}=0 \\
& f_{\infty}^{\prime}=\alpha_{H} \\
& f_{\infty}^{\prime \prime}=0
\end{aligned}
$$

The quantities $\mathrm{H}_{t w}, \mathrm{H}_{t_{\infty}}, \mathrm{K}_{i w}$, and $\mathrm{K}_{i \infty}$, are specified functions of $\mathcal{f}$. INTEGRAI WATRIX PROCEDURE: Following the method of Kendall it Bartlett ${ }^{(10)}$ the transformed equations are put into integral matrix form. The details of this procedure are presented only for the momentum equation. The handling of the species concentration and energy equations is completely analogous and is omitted in order to avoid needless algebraic complexity. 
The transformed momentum equation is:

$$
\begin{aligned}
& f f^{\prime \prime}+\left[\frac{c f^{\prime \prime}}{\alpha_{H}}\right]^{\prime}+\beta\left(\alpha_{H}^{2} \frac{P_{\infty}}{\rho}-f^{\prime 2}\right) \\
& =2\left(f^{\prime} \frac{\partial f^{\prime}}{\partial \ln \xi}-f^{\prime \prime} \frac{\partial f}{\partial \ln \xi}-f^{\prime 2} \frac{d \ln \alpha_{H}}{\partial \ln \xi}\right)
\end{aligned}
$$

The boundary layer is divided into $\mathrm{N}-1$ strips at a given streamwise station $s$. These strips are bounded by $N$ nodal points. In the final numerical solution $N=7$ was used. The points are designated $\eta_{n}$ where $n=1$ at the wall and $n=N$ on the boundary layer edge.

Let $P_{n}$ represent any of the quantities $f\left(\xi_{n}\right), f^{\prime}\left(k_{n}\right)$, f' $\left(\eta_{n}\right)$, or f'l' $\left(\eta_{n}\right), P_{n+1}$ can be related to $P_{n}$ by means of a Taylor series expansion about $\eta_{n}$ :

$$
P_{n+1}=P_{n}+P_{n}^{\prime} \delta h+P_{n}^{\prime \prime} \frac{(\delta \xi)^{2}}{2 !}+P_{n}^{\prime \prime \prime} \frac{(\delta \eta)^{3}}{3 !}+P_{n}^{\prime \prime \prime \prime} \frac{(\delta \xi)^{4}}{4 !}+\cdots
$$$$
\text { where } \delta \xi=k_{n+1}-k_{n}
$$

The highest order derivative of the dependent variable $f$ which appears in the boundary layer equations is f'l'. Therefore, the series is truncated at the next hichest derivative, fll'l. The quantity f'l'l is considered to be constant between $\eta_{n}$ and $\eta_{n+1}$.

$$
f^{\prime \prime \prime \prime}=\frac{f_{n+1}^{\prime \prime \prime}-f_{n}^{\prime \prime \prime}}{\delta \eta}
$$


Therefore the truncated Taylor series expansions for $f_{n}$, $f_{n}$ ' $f^{\prime \prime} n$ become:

$$
\begin{aligned}
& f_{n+1}=f_{n}+f_{n}^{\prime} \delta \xi+f_{n}^{\prime \prime} \frac{(\delta \xi)^{2}}{2 !}+f_{n}^{\prime \prime \prime} \frac{(\delta \xi)^{3}}{8}+f_{n+1}^{\prime \prime \prime} \frac{(\delta \xi)^{3}}{24} \\
& f_{n+1}^{\prime}=f_{n}^{\prime}+f_{n}^{\prime \prime} \delta \xi+f_{n}^{\prime \prime \prime} \frac{(\delta \xi)^{2}}{3}+f_{n+1}^{\prime \prime \prime} \frac{(\delta \xi)^{2}}{6} \\
& f_{n+1}^{\prime \prime}=f_{n}^{\prime \prime}+f_{n}^{\prime \prime \prime} \frac{\delta \xi}{2}+f_{n+1}^{\prime \prime \prime} \frac{\delta \xi}{2}
\end{aligned}
$$

The foregoing is an implicit set of relations giving $f_{n}+1$, $f^{\prime} n+1$, f' $n+1$, and $f^{\prime \prime \prime} n+1$ as functions of $\eta$. This is known as a "spline fit" and serves the same purpose as finite difference relations in usual numerical analyses. However, in this case the functions join in a continuous manner at the nodal points, thus requiring fewer nodes for the same relative accuracy. The variables $H_{t}$ and $K_{i}$ are treated in the same manner.

Derivatives in the streamrise direction are represented by the conventional three-point backward difference relations:

$$
2\left[\frac{\partial P}{\partial \ln \xi}\right]=d_{0} P_{l}+d_{1} P_{l-1}+d_{2} P_{l-2}
$$

where

$$
\left.\begin{array}{l}
d_{0}=2\left(l \Delta_{l-1}+{ }_{l} \Delta_{l-2}\right) /\left(l \Delta_{l-1} \Delta_{l-2}\right) \\
d_{1}=-2\left(\Delta_{l-2}\right) /\left(l \Delta_{l-1} \Delta_{l-1} \Delta_{l-2}\right) \\
d_{2}=2\left(l \Delta_{l-1}\right) /\left(l \Delta_{l-2} \Delta_{l-1} \Delta_{l-2}\right)
\end{array}\right\}
$$

where $P_{I}$ is any dependent variable and. $P_{I-1}$ is the variable evaluated at the previous streamwise station.

$$
{ }_{l} \Delta_{l-1}=\ln \left(\xi_{l} / \xi_{l-1}\right)
$$


12

The momentum equation can now be integrated at constant $\rho$ across each strip to obtain equations of the form:

$$
\begin{gathered}
\int_{n-1}^{n} f f^{\prime \prime} d h+\left[\frac{c f^{\prime \prime}}{\alpha_{H}}\right]_{n-1}^{n}+\beta \alpha_{H}^{2} \int_{n-1}^{n} \frac{p_{\infty}}{\rho} d h-\beta \int_{n-1}^{n} f^{\prime 2} d h \\
=\int_{n-1}^{n}\left(f^{\prime} \frac{\partial f^{\prime}}{\partial \ln \xi}-f^{\prime \prime} \frac{\partial f}{\partial \ln \xi}\right) d h-2 \int_{n-1}^{n} f^{\prime 2} \frac{d \ln \alpha_{H}}{d \ln \xi} d h
\end{gathered}
$$

The integral $\int_{n-1}^{n} f f^{\prime \prime} d \eta \quad$ can be integrated by parts to give:

$$
\int_{n=1}^{n} f f^{\prime \prime} d \xi=\left[f f^{\prime}\right]_{n-1}^{n}-\int_{n-1}^{n} f^{\prime 2} d h
$$

The remaining integral is expanded in a Taylor series.

$$
\begin{aligned}
\int_{n-1}^{n} f^{\prime} p d h= & f_{n}^{\prime} p \delta \xi-\left(f_{n}^{\prime} p^{\prime}+f_{n}^{\prime \prime} p\right) \frac{(\delta \xi)^{2}}{2 !} \\
& +\left(f_{n}^{\prime} p^{\prime \prime}+2 f_{n}^{\prime \prime} p^{\prime}+f_{n}^{\prime \prime \prime} p\right) \frac{(\delta h)^{3}}{3 !} \\
& -\left(f_{n}^{\prime} p^{\prime \prime \prime}+3 f_{n}^{\prime \prime} p^{\prime \prime}+3 f_{n}^{\prime \prime \prime} p^{\prime}+f_{n}^{\prime \prime \prime \prime} p\right) \frac{(\delta \xi)^{4}}{4 !} \\
& +\left(4 f_{n}^{\prime \prime} p^{\prime \prime \prime}+6 f_{n}^{\prime \prime \prime} p^{\prime \prime}+4 f_{n}^{\prime \prime \prime \prime} p\right) \frac{(5 \xi)^{5}}{5 !} \\
& -\left(10 f_{n}^{\prime \prime \prime} p^{\prime \prime \prime}+10 f_{n}^{\prime \prime \prime \prime} p^{\prime \prime}\right) \frac{(\delta \xi)^{6}}{6 !}+20 f_{n}^{\prime \prime \prime} p^{\prime \prime \prime} \frac{(\delta \xi)^{7}}{7 !}
\end{aligned}
$$

where $P$ may be any dependent variable. The same truncation criterion has been applied as in previous Taylor series expansions. Using the formula (38) with $P=f^{\prime}$, the integral becomes:

$$
\int_{n-1}^{n} f^{\prime 2} d \xi=f_{n}^{\prime} A_{1}+f_{n}^{\prime \prime} A_{2}+f_{n}^{\prime \prime \prime} A_{3}+f_{n-1}^{\prime \prime \prime} A_{4}
$$

where

$$
\left.\begin{array}{l}
A_{1}=\delta \xi\left(f_{n}^{\prime}-f_{n}^{\prime \prime} \frac{\delta \xi}{2}+f_{n}^{\prime \prime \prime} \frac{(\delta \xi)^{2}}{8}+f_{n-1}^{\prime \prime \prime} \frac{(\delta \xi)^{2}}{24}\right) \\
A_{2}=-(\delta \xi)^{2}\left[\frac{f_{n}^{\prime}}{2}-f_{n}^{\prime \prime} \frac{\delta \xi}{3}+f_{n}^{\prime \prime \prime} \frac{11(\delta \xi)^{2}}{120}+f_{n-1}^{\prime \prime \prime} \frac{(\delta \xi)^{2}}{30}\right]
\end{array}\right\}(40)
$$


13

$$
\left.\begin{array}{l}
A_{3}=(\delta \xi)^{3}\left[\frac{f_{n}^{\prime}}{8}-f_{n}^{\prime \prime} \frac{11 \delta \xi}{120}-f_{n}^{\prime \prime \prime} \frac{(\delta \xi)^{2}}{420}+f_{n-1}^{\prime \prime \prime} \frac{5(\delta \xi)^{2}}{504}\right] \\
A_{4}=(\delta \xi)^{3}\left[\frac{f_{n}^{\prime}}{24}-f_{n}^{\prime \prime} \frac{\delta \xi}{30}+f_{n}^{\prime \prime \prime} \frac{5(\delta \xi)^{2}}{504}+f_{n-1}^{\prime \prime \prime} \frac{(\delta \xi)^{2}}{252}\right]
\end{array}\right\}(40)
$$

Now consider the integrals of the streamwise derivatives using the finite difference approximation.

$$
\begin{aligned}
2 \int_{n-1}^{n}\left(f^{\prime} \frac{\partial f^{\prime}}{\partial \ln \xi}-f^{\prime \prime} \frac{\partial f}{\partial \ln \xi}\right) d \eta & =\int_{n-1}^{n} f^{\prime}\left(d_{0} f_{l}^{\prime}+d_{1} f_{l-1}^{\prime}+d_{2} f_{l-2}^{\prime}\right) d h \\
& -\int_{n-1}^{n} f^{\prime \prime}\left(d_{0} f_{l}+d_{1} f_{l-1}+d_{2} f_{l-2}\right) d \xi
\end{aligned}
$$

Integrating the second integral on the right hand side by parts yields:

$$
\begin{aligned}
& \int_{n-1}^{n} f^{\prime \prime}\left(d_{0} f_{l}+d_{1} f_{l-1}+d_{2} f_{l-2}\right) d h=2 d_{0} \int_{n-1}^{n} f^{\prime 2} d h \\
& +d_{1} \int_{n-1}^{n} f^{\prime} f_{l-1}^{\prime} d h+d_{1} \int_{n-1}^{n} f_{l-1}^{\prime} f^{\prime} d \xi+d_{2} \int_{n-1}^{n} f^{\prime} f_{l-2}^{\prime} d h \\
& +d_{2} \int_{n-1}^{n} f_{l-2}^{\prime} f^{\prime} d \xi-\left[d_{0} f_{l} f^{\prime}+d_{1} f_{l-1} f^{\prime}+d_{2} f_{l-2} f^{\prime}\right]_{n-1}^{n}
\end{aligned}
$$

These integrals are of the same form as equation (38) and can be expanded in a Taylor series in like manner with the result:

$$
\begin{aligned}
2 \int_{n-1}^{n}\left[f^{\prime} \frac{\partial f^{\prime}}{\partial \ln \xi}-\right. & \left.f^{\prime \prime} \frac{\partial f}{\partial \ln \xi}\right] d \xi=\left[d_{0} f_{l} f^{\prime}+d_{1} f_{l-1} f^{\prime}+d_{2} f_{l-2} f^{\prime}\right]_{n-1}^{n} \\
& +2 d_{0}\left[f_{n}^{\prime} A_{1}+f_{n}^{\prime \prime} A_{2}+f_{n}^{\prime \prime \prime} A_{3}+f_{n-1}^{\prime \prime \prime} A_{4}\right] \\
& +2\left[f_{n}^{\prime} B_{1}+f_{n}^{\prime \prime} B_{2}+f_{n}^{\prime \prime \prime} B_{3}+f_{n-1}^{\prime \prime \prime} B_{4}\right]
\end{aligned}
$$

where

$$
\left.\begin{array}{l}
B_{1}=\delta \xi\left[c_{1}-c_{2} \frac{\delta \xi}{2}+c_{3} \frac{(\delta \xi)^{2}}{8}+c_{4} \frac{(\delta \xi)^{2}}{24}\right] \\
B_{2}=-(\delta \xi)^{2}\left[\frac{c_{1}}{2}-c_{2} \frac{\delta \xi}{3}+c_{3} \frac{11(\delta \xi)^{2}}{120}+c_{4} \frac{(\delta \xi)^{2}}{30}\right] \\
B_{3}=(\delta \xi)^{3}\left[\frac{c_{1}}{8}-c_{2} \frac{11 \delta \xi}{120}+c_{3} \frac{11(\delta \xi)^{2}}{420}+c_{4} \frac{5(\delta \xi)^{2}}{504}\right]
\end{array}\right\}
$$




$$
B_{4}=(\delta \xi)^{3}\left[\frac{c_{1}}{24}-c_{2} \frac{\delta \xi}{30}+c_{3} \frac{5(\delta 4)^{2}}{504}+c_{4} \frac{(\delta \xi)^{2}}{252}\right]
$$

and

$$
\begin{aligned}
& c_{1}=d_{1} f_{l-1, n}^{\prime}+d_{2} f_{l-2, n}^{\prime} \\
& c_{2}=d_{1} f_{l-1, n}^{\prime \prime}+d_{2} f_{l-2, n}^{\prime \prime} \\
& c_{3}=d_{1} f_{l-1, n}^{\prime \prime \prime}+d_{2} f_{l-2, n}^{\prime \prime \prime} \\
& c_{4}=d_{1} f_{l-1, n-1}^{\prime \prime \prime}+d_{2} f_{l-2, n-1}^{\prime \prime \prime}
\end{aligned}
$$

Finally, a similar procedure applied to the third streamvise integral yields:

$$
\begin{aligned}
& 2 \int_{n-1}^{n} f^{\prime 2} \frac{d \ln \alpha_{H}}{d \ln \xi} d \eta=\left(d_{0}+\frac{d_{1} \alpha_{H \ell-1}+d_{2} \alpha_{H \ell-2}}{\alpha_{H}}\right) \times \\
& {\left[f_{n}^{\prime} A_{1}+f_{n}^{\prime \prime} A_{2}+f_{n}^{\prime \prime \prime} A_{3}+f_{n-1}^{\prime \prime \prime} A_{A}\right] }
\end{aligned}
$$

It remains to evaluate $\int_{n=1}^{n} \frac{\rho_{\infty}}{\rho} d h$. This can be done by approximating $\frac{\rho_{\infty}}{\rho}$ as a cubic between $n-1$ and $n$. The four coefficients are evaluated by matching the cubic aproximation to the value of $\frac{P_{\infty}}{P}$ and $\left(\frac{P_{\infty}}{\rho}\right)^{\prime}$ at adjacent nodal points. The function can then be integrated exactly across the strip to give:

$$
\int_{n-1}^{n} \frac{P_{\infty}}{\rho} d \xi=\left(\frac{P_{\infty}}{P_{n}}+\frac{P_{\infty}}{P_{n-1}}\right) \frac{\delta \xi}{2}+\left(\frac{P_{\infty} P_{n}^{\prime}}{P_{n}^{2}}-\frac{P_{\infty} P_{n-1}^{\prime}}{P_{n-1}^{2}}\right) \frac{(\delta \xi)^{2}}{12}
$$

This approximation will not be quite as accurate as the preceding ones since the derivatives are not necessarily continuous at each node. 
Substituting the previously derived formulas into the momentum equation yields:

$$
\begin{aligned}
& {\left[\frac{c f^{\prime \prime}}{\alpha_{H}}+f^{\prime}\left(\left(1+d_{0}\right) f+d_{1} f_{l-1}+d_{2} f_{l-2}\right)\right]_{n-1}^{n}} \\
& +\beta \alpha_{H}^{2}\left[\left(\frac{\rho_{\infty}}{P_{n}}+\frac{\rho_{\infty}}{\rho_{n-1}}\right) \frac{\delta \xi}{2}+\left(\frac{\rho_{\infty} P_{n}^{\prime}}{\rho_{n}^{2}}-\frac{\rho_{\infty} P_{n-1}^{\prime}}{\rho_{n-1}^{2}}\right) \frac{(\delta \xi)^{2}}{12}\right] \\
& -\left(1+\beta+d_{0}-\frac{d_{1} \alpha_{H e-1}+d_{2} \alpha_{H 2-2}}{\alpha_{H}}\right) \times\left[f_{n}^{\prime} A_{1}+f_{n}^{\prime \prime} A_{2}+f_{n}^{\prime \prime \prime} A_{3}\right. \\
& \left.+f_{n-1}^{\prime \prime \prime} A_{4}\right]-2\left[f_{n}^{\prime} B_{1}+f_{n}^{\prime \prime} B_{2}+f_{n}^{\prime \prime \prime} B_{3}+f_{n-1}^{\prime \prime \prime} B_{4}\right]=0
\end{aligned}
$$

The real power of the integral-matrix method comes from the fact that integrals of functions rather than the functions themselves and their derivatives are approximated. Since integrals tend to be mach smoother functions, the approximations give much more accurate results than can be attained by approximating the functions directly. 
MATRIX SOLUTIONS PROCEDURE: A completely analogous procedure is applied to the species conservation and energy equations. The resulting matrix of equations contains $3(\mathrm{~N}-1)$ conservation equations over the $\mathbb{N}-1$ intervals, $7(N-1)$ Taylor series expansions, 10 boundary conditions, and the $\alpha_{H}$ constraint. These contain $10 \mathrm{~N}+1$ variables, i.e., the primary variables $f, H_{t}$, and $K_{i}$ and their derivatives at each nodal point and $\alpha_{H}$. These equations are solved through use of a generalized Newton-Raphson iteration procedure.

This can best be illustrated for the arbitrary functions $F_{1}$ and $F_{2}$. Suppose $F_{1}(x, y)$ and $F_{2}(x, y)$ are complicated functions of $x$ and $y$. Further suppose $\bar{x}, \bar{y}$ represent the solution to the equations $F_{1}=0$, $F_{2}$. One can expand the functions in a Taylor series to first order terms.

$$
\left.\begin{array}{l}
F_{1}(\bar{x}, \bar{y})=0=F_{1}\left(x_{m}, y_{m}\right)+\left(\bar{x}-x_{m}\right) \frac{\partial F_{1}\left(x_{m}, y_{m}\right)}{\partial x}+\left(\bar{y}-y_{m}\right) \frac{\partial F_{1}\left(x_{m}, y_{m}\right)}{\partial y} \\
F_{2}(\bar{x}, \bar{y})=0=F_{2}\left(x_{m}, y_{m}\right)+\left(\bar{x}-x_{m}\right) \frac{\partial F_{2}\left(x_{m}, y_{m}\right)}{\partial x}+\left(\bar{y}-y_{m}\right) \frac{\partial F_{2}\left(x_{m}, y_{m}\right)}{\partial y}
\end{array}\right\}\left(4 y^{\prime}\right)
$$

where $x_{m}, y_{m}$ are the values of $x$ and $y$ after the mth iteration. If $\bar{x}, \bar{y}$ are replaced by $x_{m+1}, y_{m+1}$, the equations (49) yield:

where

$$
\begin{aligned}
& \left.\Delta x_{m} \frac{\partial F_{1}}{\partial x}\right|_{m}+\left.\Delta y_{m} \frac{\partial F_{1}}{\partial y}\right|_{m}=-F_{1 m} \\
& \left.\Delta x_{m} \frac{\partial F_{2}}{\partial x}\right|_{m}+\left.\Delta y_{m} \frac{\partial F_{2}}{\partial y}\right|_{m}=-F_{2 m}
\end{aligned}
$$

$$
\begin{aligned}
& \Delta x_{m}=x_{m+1}-x_{m} \\
& \Delta y_{m}=y_{m+1}-y_{m}
\end{aligned}
$$

and the subscript $m$ denotes the quantity evaluated at $x_{m}, y_{m}$ 
In matrix notation,

$$
\left[\begin{array}{ll}
\left.\frac{\partial F_{1}}{\partial x}\right|_{m} & \left.\frac{\partial F_{1}}{\partial y}\right|_{m} \\
\left.\frac{\partial F_{2}}{\partial x}\right|_{m} & \left.\frac{\partial F_{2}}{\partial y}\right|_{m}
\end{array}\right]^{\Delta x_{m}}\left[\begin{array}{l}
\Delta Y_{m}
\end{array}\right]=\left[\begin{array}{l}
-F_{1 m} \\
-F_{2 m}
\end{array}\right]
$$

Notice that the coefficient matrix and the right hand side are functions of $\mathrm{x}_{\mathrm{m}}, \mathrm{y}_{\mathrm{m}}$ only. Therefore, $\Delta \mathrm{x}_{\mathrm{m}}, \Delta \mathrm{y}_{\mathrm{m}}$ can be calculated in terms of $F_{1}$ and $F_{2}$ and their derivatives evaluated at $x_{m}, y_{m} \cdot$

$$
\left[\begin{array}{l}
\Delta x_{m} \\
\Delta y_{m}
\end{array}\right]=\left[\begin{array}{ll}
\left.\frac{\partial F_{1}}{\partial x}\right|_{m} & \left.\frac{\partial F_{1}}{\partial y}\right|_{m} \\
\left.\frac{\partial F_{2}}{\partial x}\right|_{m} & \left.\frac{\partial F_{2}}{\partial y}\right|_{m}
\end{array}\right]^{-1}\left[\begin{array}{l}
-F_{1 m} \\
-F_{2 m}
\end{array}\right]
$$

The quantities $\Delta \mathrm{x}_{\mathrm{m}}, \Delta \mathrm{y}_{\mathrm{m}}$ represent corrections to be added to $\mathrm{x}_{\mathrm{m}}, \mathrm{y}_{\mathrm{m}}$ to give new values of the dependent variables $x_{m+1}, y_{m+1}$. A solution is obtained by guessing $x_{1}, y_{1}$, calculating the corrections, obtaining $\mathrm{x}_{2}, \mathrm{y}_{2}$, etc. The procedure continues until $\mathrm{F}_{1}$ and $\mathrm{F}_{2}$ approach zero or until the corrections become arbitrarily small depending on accuracy requirements.

Obviously this procedure may be extended to any number of variables and empations. This has been done in the present study with excellent results. 
The linearized momentum equation becomes:

$$
\begin{aligned}
& {\left[\frac{c f^{\prime \prime}}{\alpha_{H}}\left(\frac{\Delta f^{\prime \prime}}{f^{\prime \prime}}+\frac{\Delta c}{c}-\frac{\Delta \alpha_{H}}{\alpha_{H}}\right)+\left(\left(1+d_{0}\right) f+d_{l} f_{l-1}+d_{2} f_{l-2}\right) \Delta f^{\prime}\right.} \\
& \left.+f^{\prime}\left(1+d_{0}\right) \Delta f\right]_{n-1}^{n}+\beta \alpha_{H}\left[\left(\frac{P_{\infty}}{P_{n}}+\frac{P_{\infty}}{P_{n-1}}\right) \delta \xi+\left(\frac{P_{\infty} P_{n}^{\prime}}{P_{n}^{2}}-\frac{P_{\infty} P_{n-1}^{\prime}}{P_{n-1}^{2}}\right) \frac{(\delta \xi)^{2}}{\sigma}\right] \Delta \alpha_{H} \\
& -\beta \alpha_{H}^{2} \frac{\rho_{\infty}}{\rho_{n}^{2}} \frac{\delta \xi}{2}\left(1+\frac{\delta \xi}{3} \frac{\rho_{n}^{\prime}}{\rho_{n}}\right) \Delta \rho_{n}-\beta \alpha_{H}^{2} \frac{\rho_{\infty}}{\rho_{n-1}^{2}} \frac{\delta \xi}{2}\left(1-\frac{\delta \xi}{3} \frac{\rho_{n-1}^{\prime}}{\rho_{n-1}}\right) \Delta \rho_{n-1} \\
& +\beta \alpha_{H}^{2} P_{\infty} \frac{(\delta \zeta)^{2}}{12}\left(\frac{\Delta P_{n}^{\prime}}{\rho_{n}^{2}}-\frac{\Delta \rho_{n-1}^{\prime}}{\rho_{n-1}^{2}}\right)-2\left[1+\beta+d_{0}-\frac{d_{1} \alpha_{H \ell-1}+d_{2} \alpha_{H \ell-2}}{\alpha_{H}}\right] x \\
& {\left[A_{1} \Delta f_{n}^{\prime}+A_{2} \Delta f_{n}^{\prime \prime}+A_{3} \Delta f_{n}^{\prime \prime \prime}+A_{4} \Delta f_{n-1}^{\prime \prime \prime}\right]} \\
& -\left(\frac{d_{1} \alpha_{H \ell-1}+d_{2} \alpha_{H \ell-2}}{\alpha_{H}^{2}}\right)\left[f_{n}^{\prime} A_{1}+f_{n}^{\prime \prime} A_{2}+f_{n}^{\prime \prime \prime} A_{3}+f_{n-1}^{\prime \prime \prime} A_{4}\right] \Delta \alpha_{H} \\
& -2\left[B_{1} \Delta f_{n}^{\prime}+B_{2} \Delta f_{n}^{\prime \prime}+B_{3} \Delta f_{n}^{\prime \prime \prime}+B_{4} \Delta f_{n-1}^{\prime \prime \prime}\right]=\text {-error }
\end{aligned}
$$

The quantity "error" is simply the momentum equation $(4,8)$ evaluated after the mth iteration. Similar expressions may be derived for the species and energy equations.

The solution for this set of equations has been programmed for digital computations. Convergence has been very rapid, usually in three to four iterations for the first streamrise station. Dormstream stations converge in one or two iterations since the upstream solution is used as a first guess for the next point downstream. An entire case including discontinuous mass injection is routinely solved for trenty streamrise stations and seven nodal points in approxinately one and one half minutes on a Univac 1108 computer. 
III. RESUITS IND DISCIISSION

Calculations have been performed for several planar bodies with air into air injection. Plates of one and five-foot lengths with both sharp and blunt leading edges have been considered. Freestream conditions were held constant for all cases with $M_{\infty}=2.0, P_{\infty}=.0428$ atm., and $\mathrm{T}_{\infty}=144_{1} 2^{\circ}$. Furthermore, only isothermal surfaces at $750^{\circ} \mathrm{R}$ were considered. Graphs are plotted with $\dot{q}_{w} \dot{q}_{\text {st }}$ as the ordinate, where $\dot{q}_{w}$ is the local heat transfer rate at the wall while $\dot{q}_{s t}$ corresponds to the heat transfer at the stagnation point for the case of no injection. In the cases of plates with sharp leading edges, $\dot{q}_{s t}$ is the stagnation point heat transfer for a geometrically similar body with a blunt leading edge. The abscissa is the distance from the leading edge of the plate. Figures 1, 2, and 3 present the results for a one-foot plate with a sharp leading edge. In each case, the injection is distributed over the first 10, 20 and $40 \%$ of the plate length while holding the total mass of injectant constant. It is observed that increasing the total mass of coolant injected in successive cases increases the magnitude of the cooling effect but leaves the shape of the curves relatively unchanged.

In the injection region, the heat transfer is observed to decrease relative to the no injection case as the distance from the leading edge decreases. This is followed by a sharp rise in heat transfer immediately downstream from the point where injection stops. Finally, the curve approaches the no injection heat transfer rate asymtotically at larger distances downstream from shere injection stops. 
The mathematical model actually predicts a discontinuous jump in heat transfer at the termination of injection. This is due to the fact that no internal heat conduction is permitted in the isothermal wall. In reality, such conduction would occur and the wall could not be held exactly at a constant temperature. The curves have been draw smoothly to a cusp as a compromise at this point.

It can be observed that the length needed for recovery to the zero injection heat transfer rate depends on the length of the injection region as well as the coolant injection rate. As the length of the injection region increases, so the distance required for recovery increases. The physical reasoning behind this fact is felt to be as follows; the persistence of any insulating effect downstream from injection is due to the presence of extra mass in the boundary layer. This mass causes both a thickening of the boundary layer and an increase in the total heat capacity of the boundary layer. Furthermore, the boundary layer naturally grows with distance from the leading edge at a rate roughly proportional to $x^{\frac{1}{2}}$ where $x$ is the distance from the leading edge. Thus, at points domstream from injection cutoff, the boundary layer will naturally be thicker and grow more slorly than at points upstream. Therefore, the extra thickening due to injection dies out more slowly at dowstream points than at points near the leading edge. In this way, the heat blockage due to injection can persist for greater distances when the injection region is Ionger.

Figures 4, 5, and 6 present similar results for a five-foot plate. Comparison of results for the one and five-foot plates shows the lack 
of any direct scaling. This is due to the highly nonlinear equations that describe the system. The boundary layer thickness does not scale directly with the geometric dimensions. Thus the five-fnot plate is more strongly affected by proportionately equal mass injection than is the one-foot plate. Figure 7 presents the same results as Figure 6 but in the form of a ratio of heat transfer with injection to heat transfer with no injection.

Figures 8 and 9 show typical velocity and temperature profiles. The profiles in the injection region show a basic difference from those downstream in that the injection region profiles exhibit a reverse curvature near the wall. Injection rates large enough to cause negative heat transfer rates usually caused the numerical solution to become unstable as profiles became nearly vertical at the wall. The actual prediction of boundary layer blowoff is not possible though since one of the basic assumptions of the laminar boundary layer equations is violated under blowoff conditions. The profiles recover their characteristic laminar shape a short distance downstream from the end of injection as the slow injected air is accelerated in the boundary layer.

Figures 10 and 12 show heat transfer rates over the leading edge of one and five-foot blunt plates. The pressure distribution over the leading edge was taken from the experimental results of Gowen and Perkins ${ }^{(14)}$. Injection vas limited to the first $22_{-5}^{\circ}$ of the circular arc. The qualitative nature of the results is the same as the sharp plate cases. The increase in heat transfer over the stagnation heat transfer at about 7$]^{\circ} \mathrm{O}$ has been observed experimentally by Rose, et. al. (15) 
This initial rise may be due to the high initial acceleration of the flow away from the stagnation point. This causes an actual thinning of the boundary layer for a short distance dormstream.

The abrupt change in slope at $15^{\circ}$ is more difficult to explain. It is not certain if this is a physical phenomenon or a numerical instability. The solution is very sensitive to the pressure gradient parameter in this region.

Figures 11 and 13 show the dornstream effects of stagnation point heat transfer. In Figures 14 and 15, the injection region extends over $75^{\circ}$ of the radius. The results are qualitatively the same as before though the magnitude of the effect is much larger. At the end of the plate, the heat transfer is still about $18 \%$ lower than the zero injection case as shom in Figure 16. 


\section{CONCLUSIONS}

The effect of upstream mass injection on downstream heat transfer in a laninar boundary layer has been investigated for supersonic flow over flat plates with both sharp and blunt leading edges. The complete set of nonsimilar boundary layer equations was solved using the integral matrix technique. In cases with large mass injection rates, an insulating effect has been found to persist for a considerable distance downstream from injection cutoff. Furthermore, the length of the injection region has also been found to have a substantial effect on the distance required for the heat blockage effects due to injection to die out. For a given total coolant mass, longer injection regions were found to require proportionally longer distances for the local heat transfer rate to approach a given percentage of the local zero injection heat transfer rate. 


\section{BIBLIOGRAPHY}

1. Low, George M.;

"The Compressible Laminar Boundary Layer with Fluid Injection", NACA TN 3404, 1955.

2. Iee, George, and Sundell, Robert E.;

"Apollo ifterbody Heat Transfer and Pressure with and without Ablation at $\mathrm{H}_{\infty}$ of 5.8 to $8.3^{\prime \prime}$, NASA TN D-3620, 1966.

3. Smith, A. M. O., and Clutter, D. W.; "Hachine Calculation of Compressible Iaminar Boundary Layers", AIAA Journal, Vol, 3, No. 4, April, 1965.

4. Rubesin, lorris W., and Inouye, Namoru;

" $\Lambda$ Theoretical Study of the Effect of Upstream Transpiration Cooling on the Heat-Transfer and Skin-Friction Characteristics of a Compressible, Laminar Boundary Layer", $\mathrm{NACA}$ TN 3969, 1957.

5. Iibby, Paul A., and Pallone, Adrian;

"A lethod for Analyzing the Heat Insulating Properties of the Laminar Compressible Boundary Layer", Journal Aero. Sci., Vol. 21, No. 12, Dec., 1954.

6. Howe, John T.;

"Some Finite Difference Solutions of the Laminar Compressible Boundary Layer Showing the Efiects of Upstream Transpiration Cooling", NASA IWISC 2-26-59A, 1959.

7. Chung, Paul K.;

"Effects of Localized liass Iransfer near the Stagnation Region of Blunt Bodies in Hypersonic Flight", NASA TN D-14I, 1960.

8. Cresci, Robert J.;

"Theoretical Analysis of the Dormstream Influence of liass Transfer in a Stagnation Region", Int. Jour. Heat Mlass Transfer, Vol. 5, pp. 837-857, September, 1962.

9. Pallone, Adrian;

"Nonsimilar Solutions of the Compressible-Laminar Boundary-Layer Equations ifth Applications to the Upstream-Transviration Cooling Problem", Jour. sero. Sci., Vol, 28, Ho. 6, June, 1961.

10. Kendall, R. N., and Bartlett, E. P.;

"Nonsimilar Solution of" the Nalticomponent Liminar Boundary Layer by an Integral-liatrix Nethod," AIAA Journal, Vol. 6, No. 6, June, 1968. 
11. Dorodnitsyn, $\Lambda$. A.;

"General liethod of Integral Relations and its hpplication to Boundary Layer Theory", Advances in Aeronautical Sciences, Vol. 3, pp. 207-219, 1960.

12. Hirschfelder, J. O., Curtis, C. F., and Bird, R. B.; liolecular Theory of Gases and Iiquids, John viiley and Sons, Inc., New York, 1954.

13. JANAF Interim Thermochemical Tables, Midland, Michigan, 1960.

14. Gowen, Forrest E., and Perkins, Edward W.; "Drag of Circular Cylinders for a Wide Range of Reynolds Numbers and líach Numbers", NACA TN 2960, 1953.

15. Kemp, Nelson H., Rose, Peter H., and Detra, Ralph W.; "Laminar lleat "Iransfer Around Blunt Bodies in Dissociated Air", Avco Research Laboratory Report 15, May, 1958. 
APPENDIX 


\section{LIST OF FIGURES}

1. Heat Transfer to a 1 - foot Sharp Plate rith Small Rates of Injection

2. Heat Transfer to a 1 - foot Sharp Plate rith Hoderate Rates of Injection

3. Heat Transfer to a 1 - foot Sharp Plate with Iarge Rates of Injection

4. Heat Transfer to a 5 - foot Sharp Plate with Small Rates of Injection

5. Heat Transfer to a 5 - foot Sharp Plate with lioderate Rates of Injection

6. Heat Transfer to a 5-foot Sharp Plate with Large Rates of Injection

7. Reduction in Heat Transfer to a 5 - foot Sharp Plate with Large Rates of Injection

8. Velocity Profiles along a 5 - foot Sharp Plate with Injection

9. Temperature Profiles along a 5 - foot Sharp Plate with Injection

10. Heat Transfer to the Leading Edge of a 1 - foot Blunt Plate

11. Downstream Heat Transfer to a 1 - foot Blunt Plate

12. Heat Transfer to the Leading Edge of a 5 - foot Blunt Plate

13. Downstream Heat Transfer to a 5 - foot Blunt Plate

14. Heat Transfer to the Leading Edge of a 1 - foot Blunt Plate

15. Dormstream Heat Transfer to a 1 - foot Blunt Plate

16. Recuction in Heat Transfer Downstream on a 1 - foot Blunt Plate with Injection in the Stagnation Region 


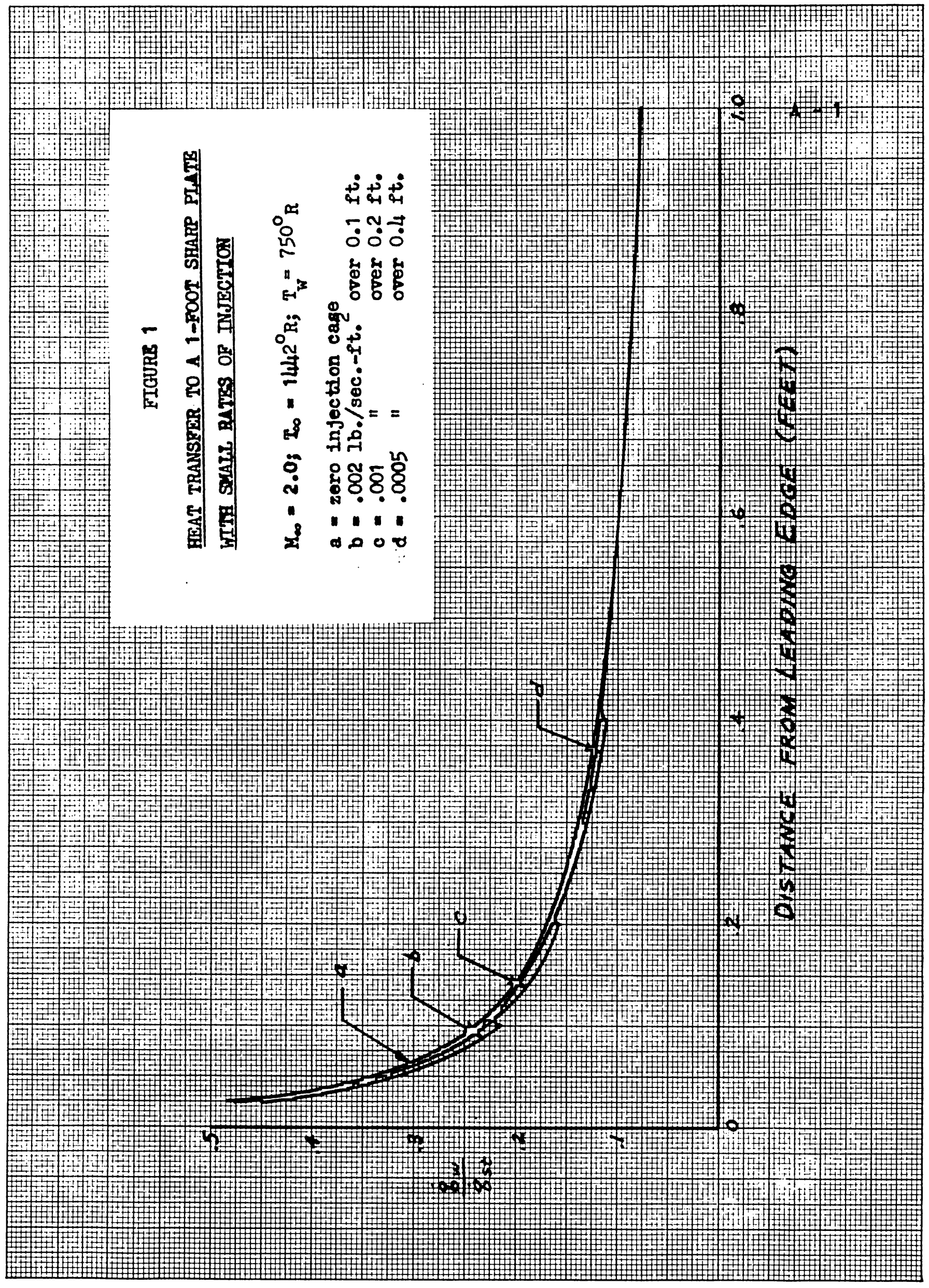




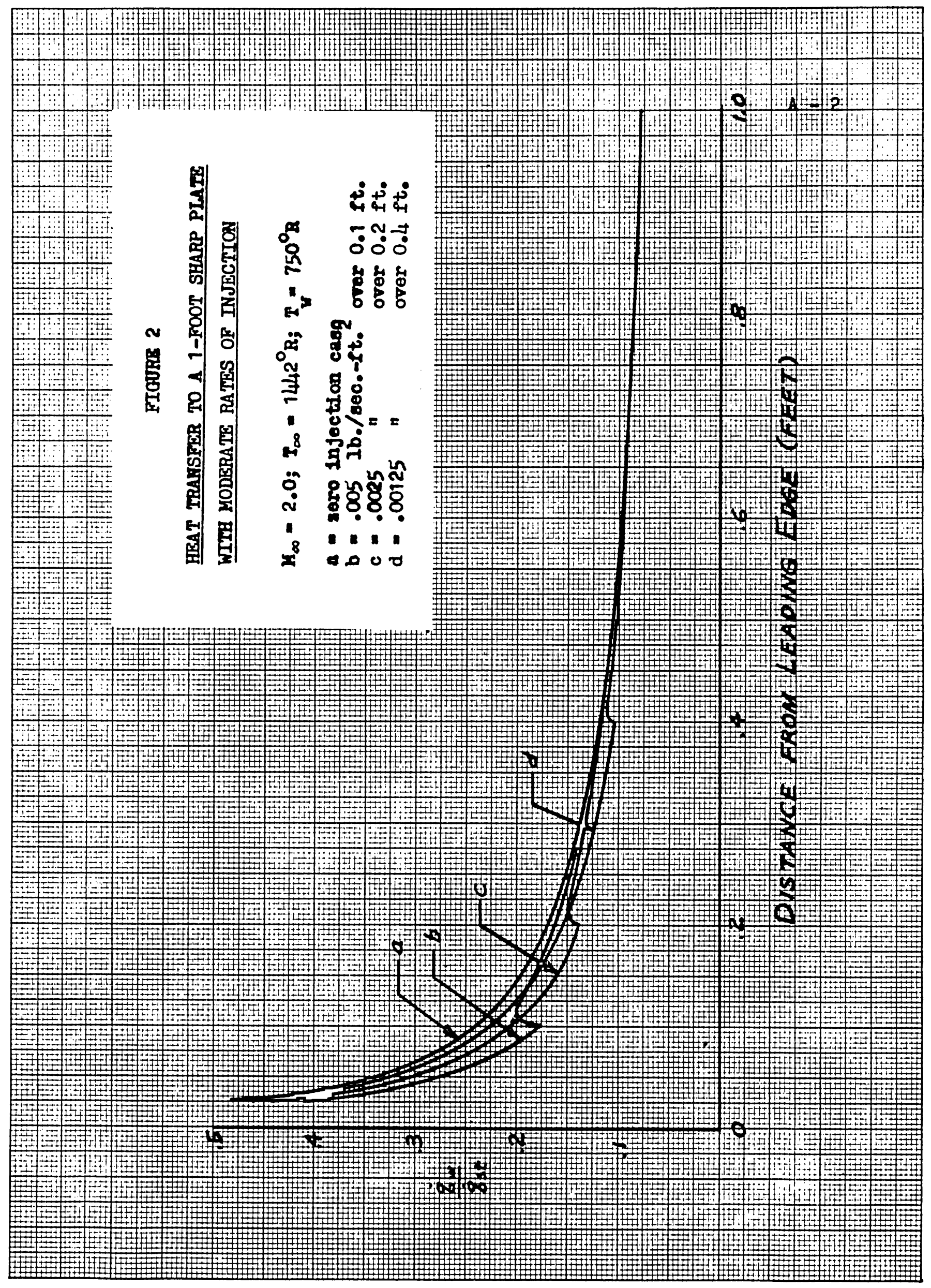




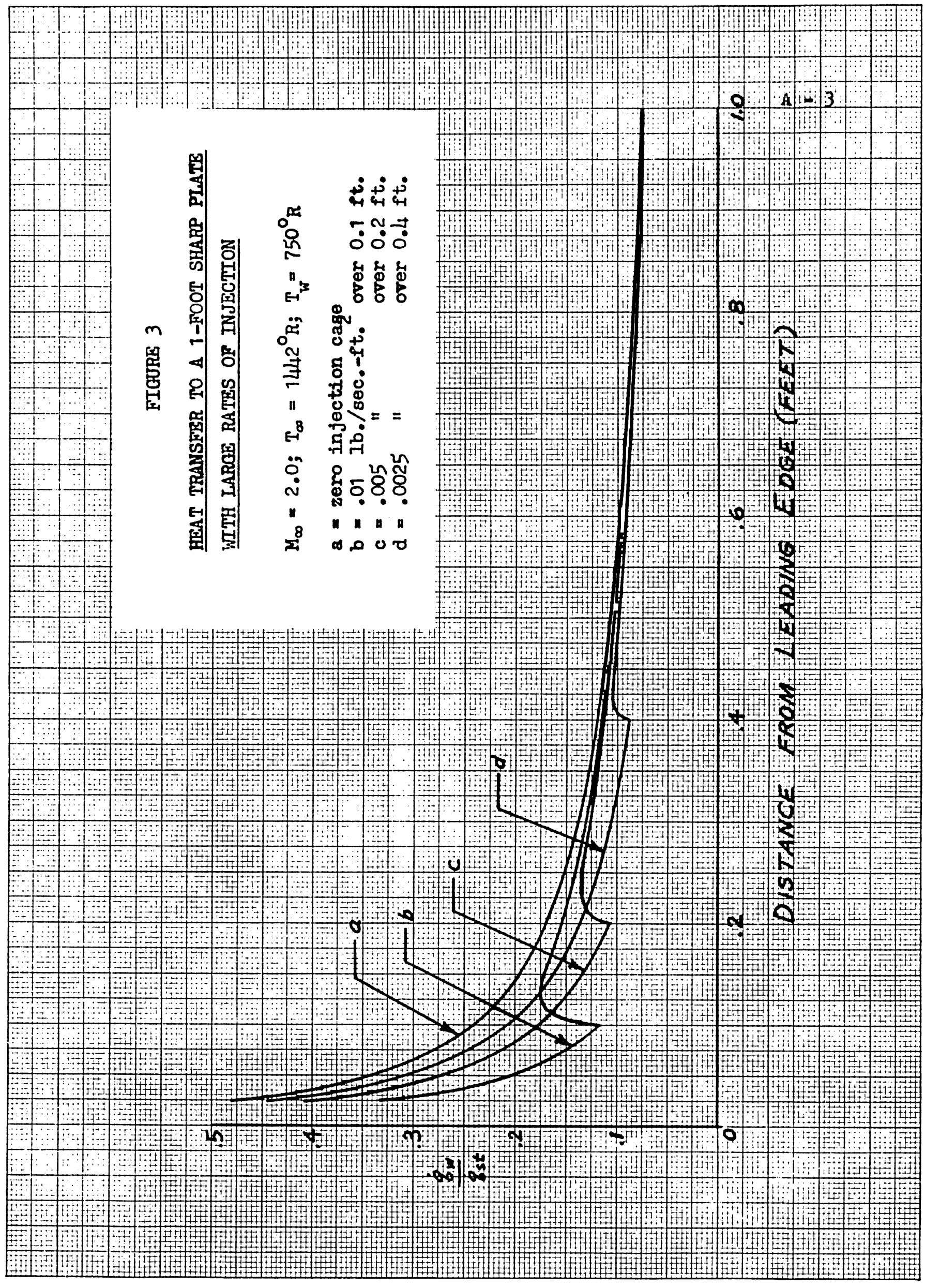




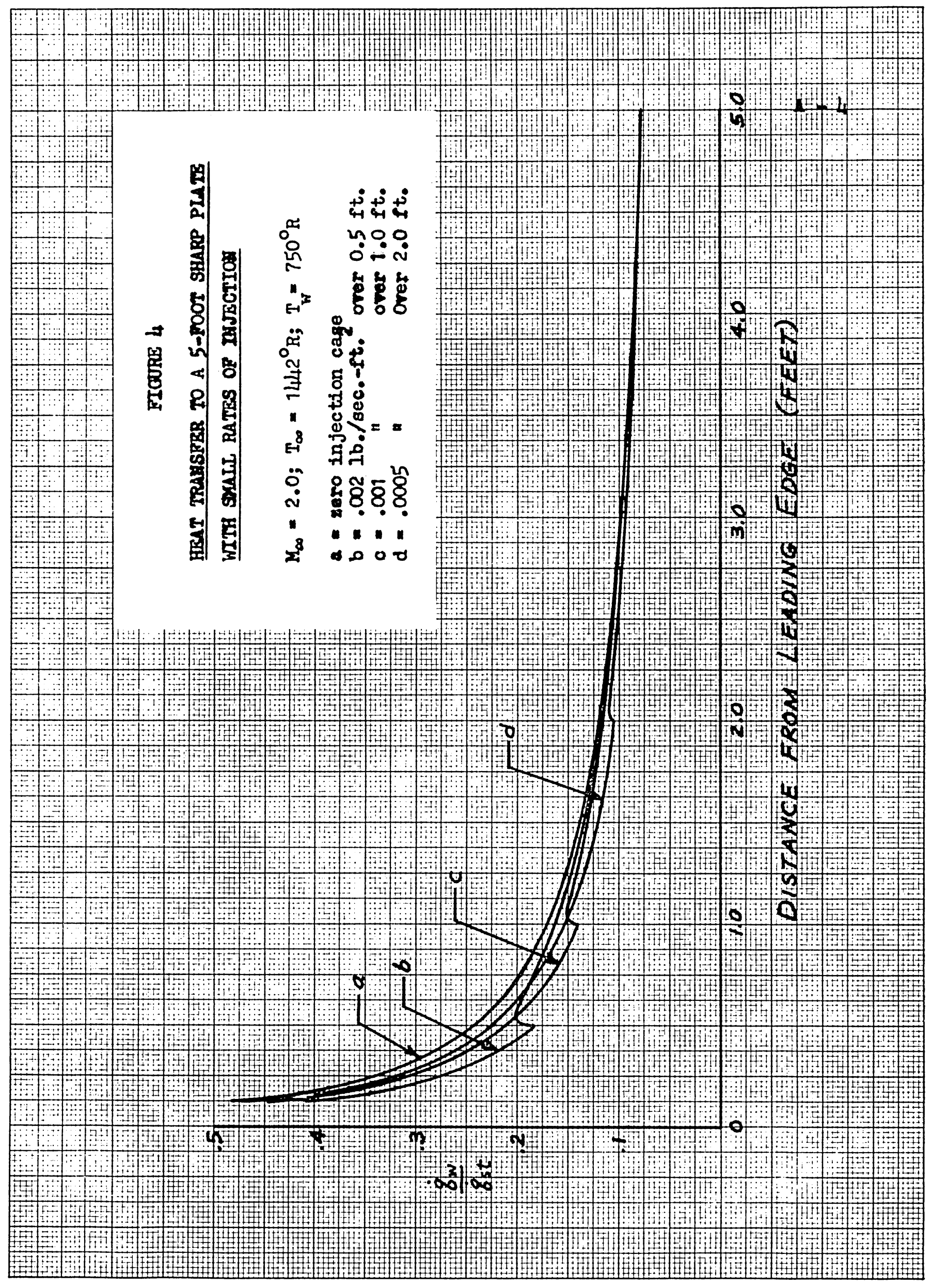




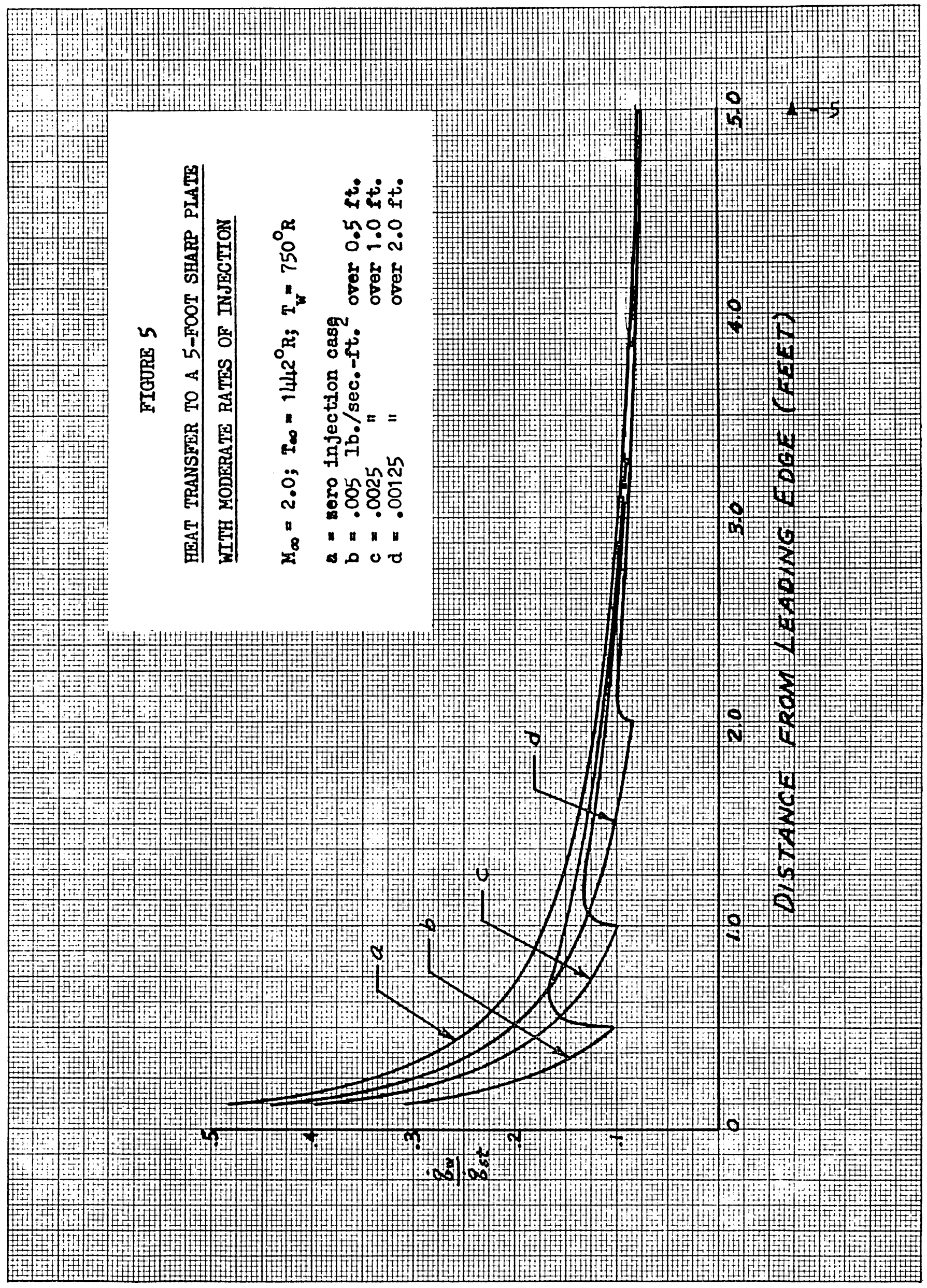




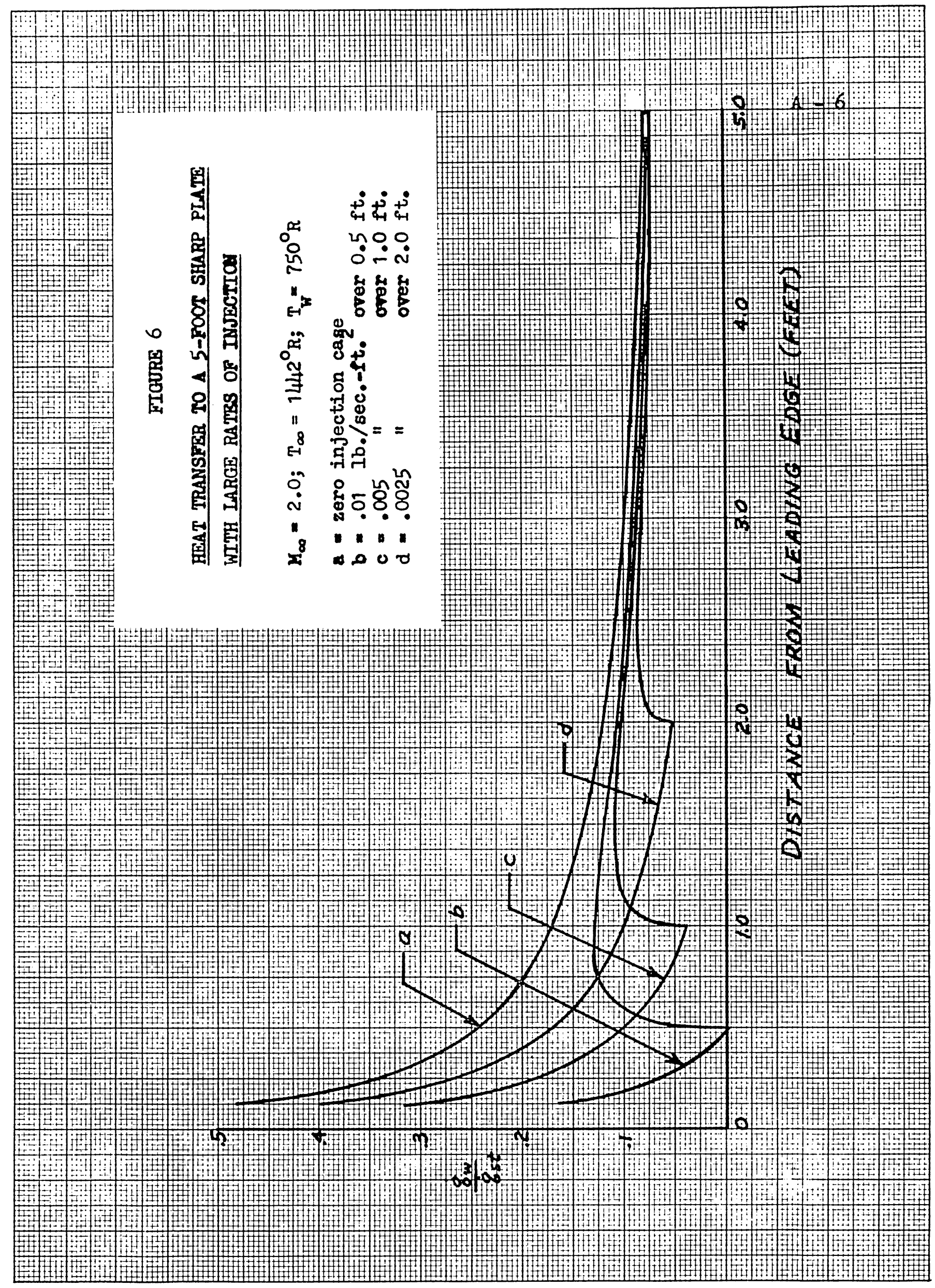




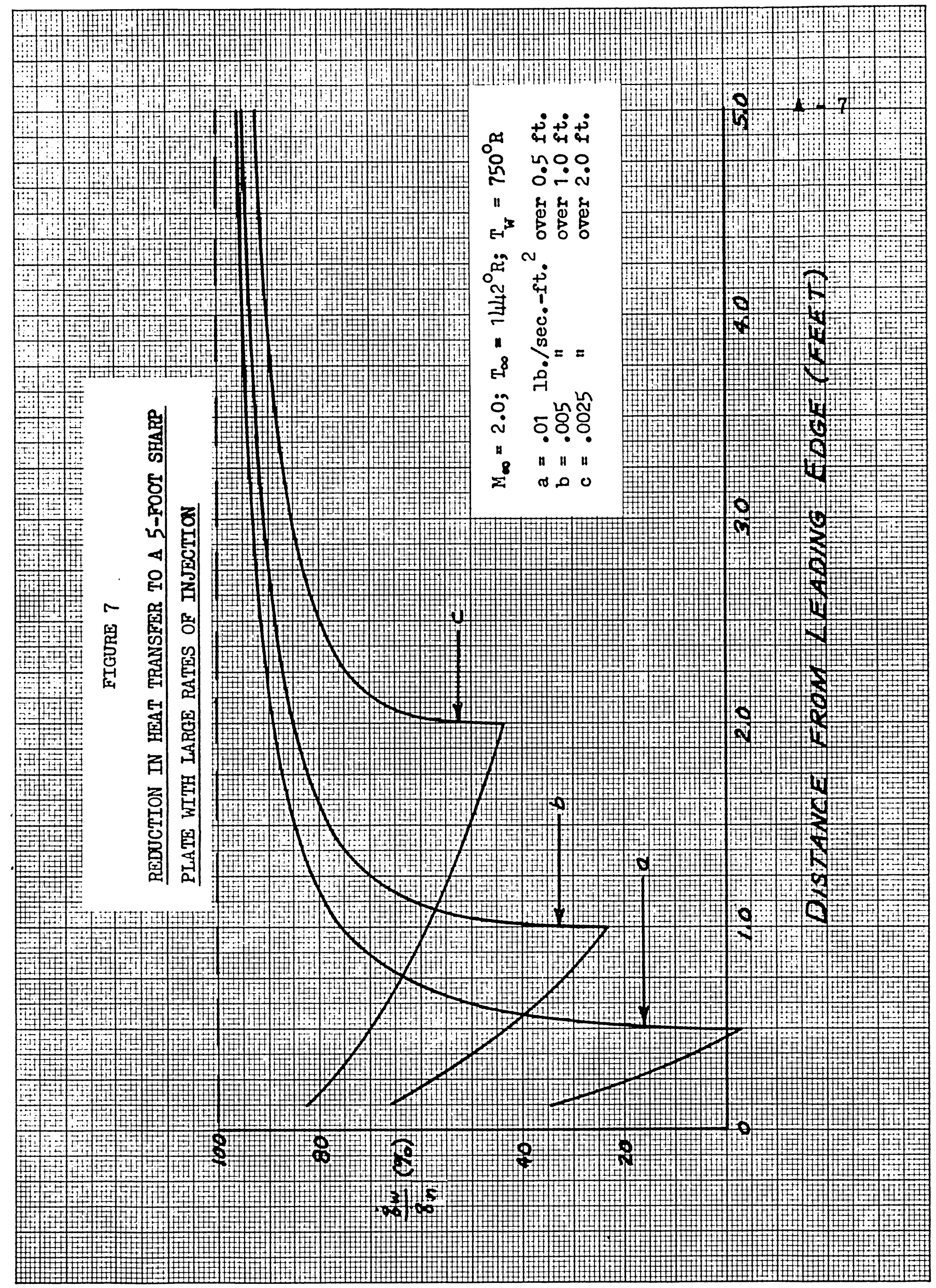




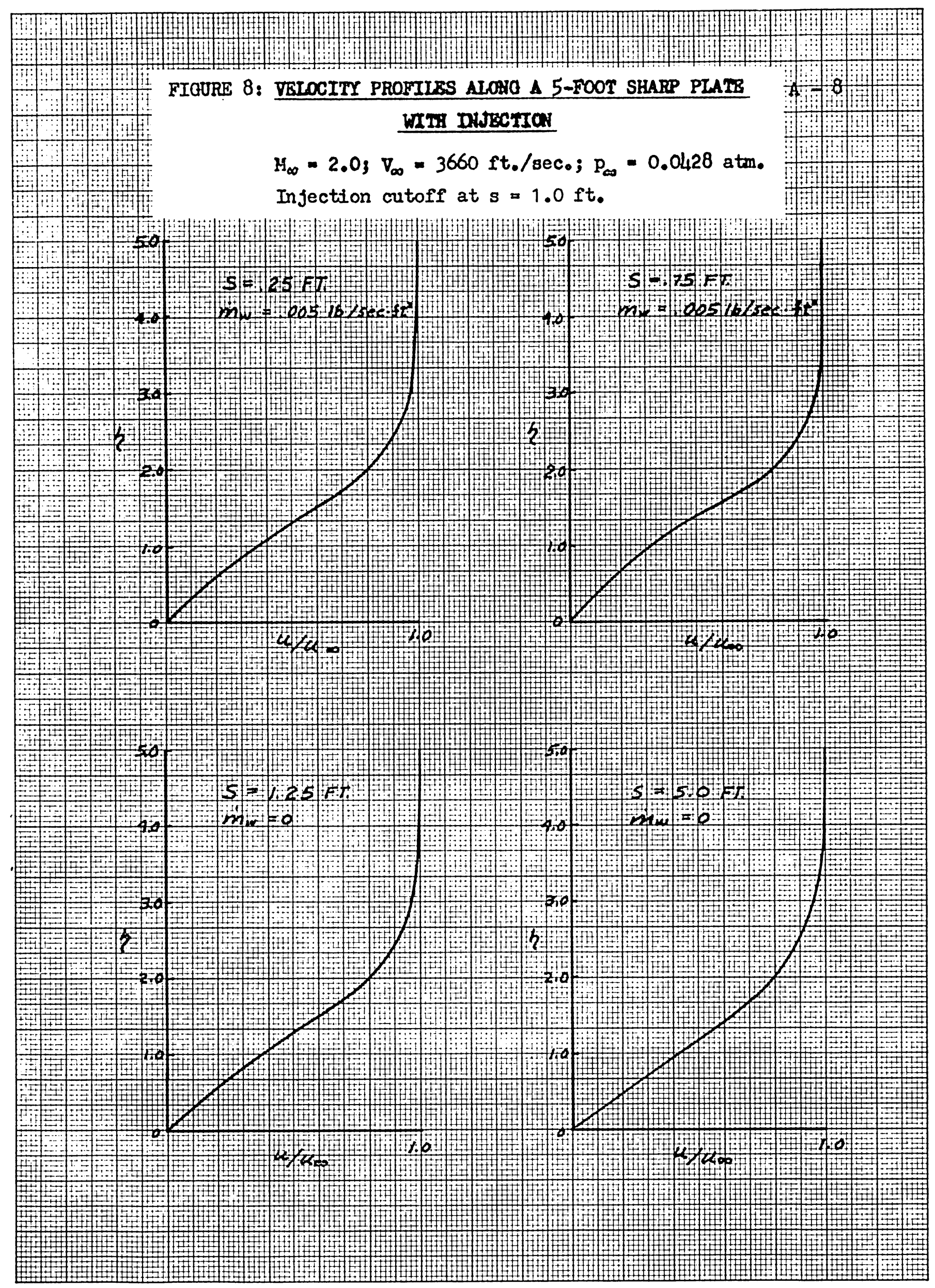




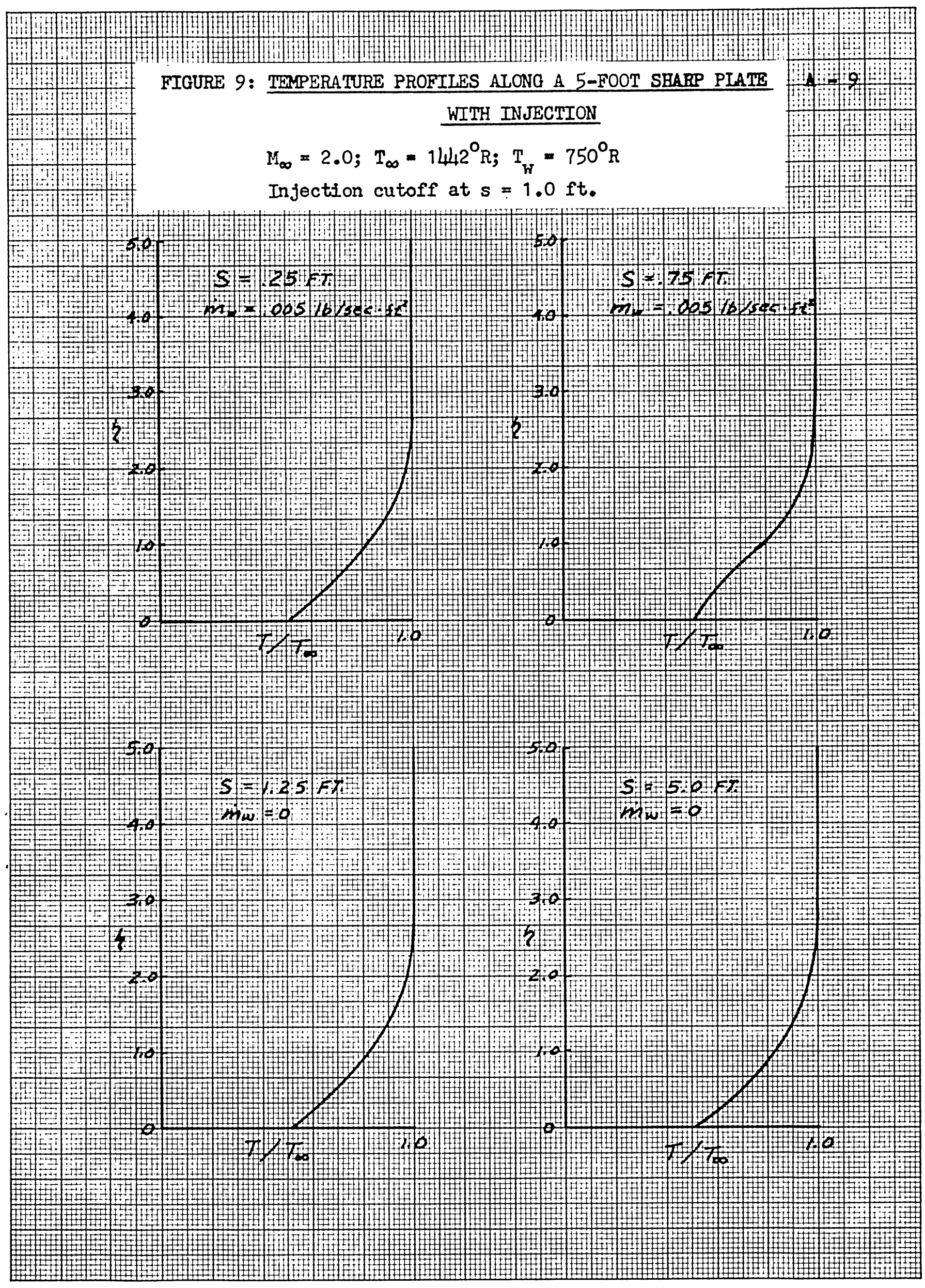




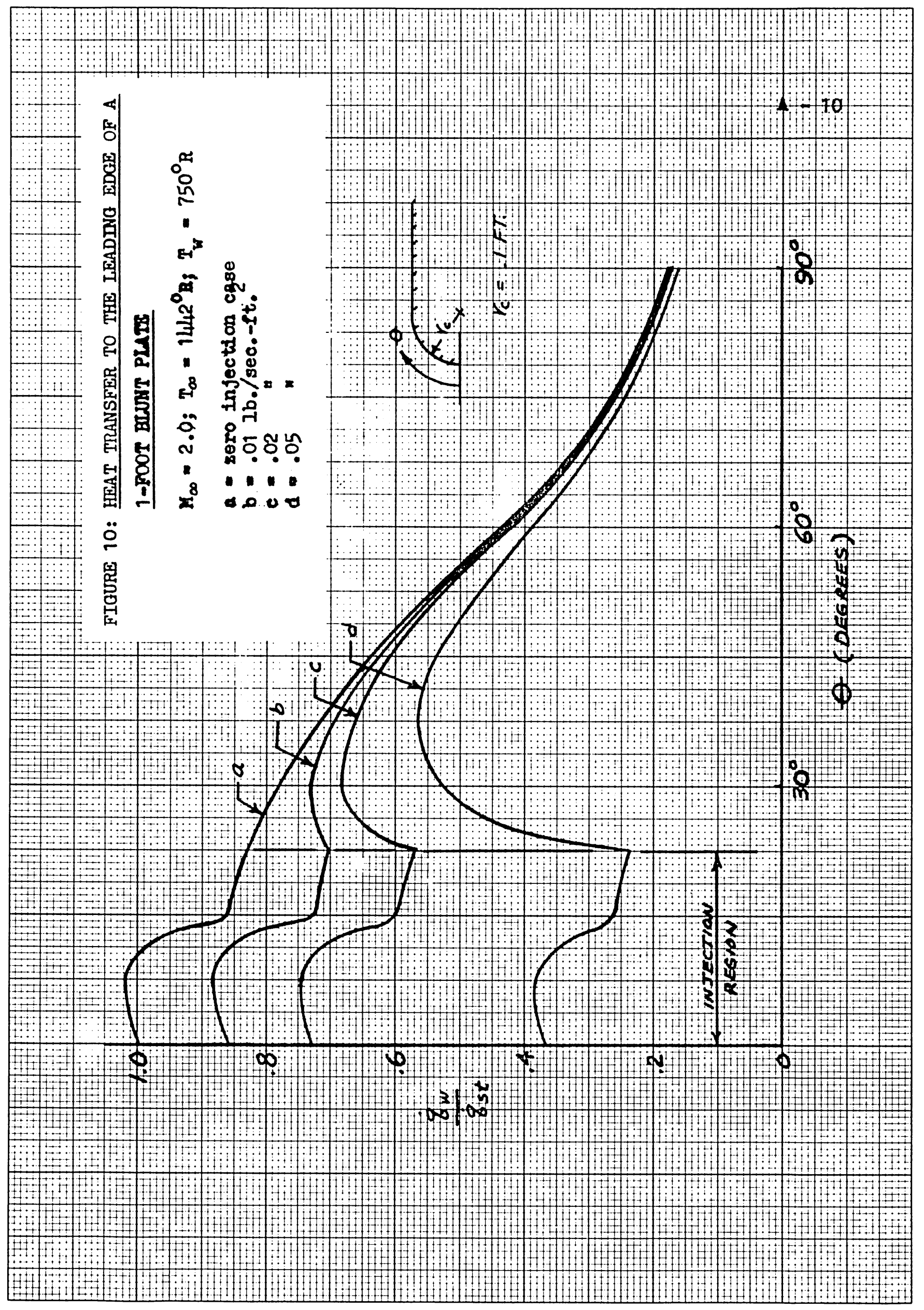




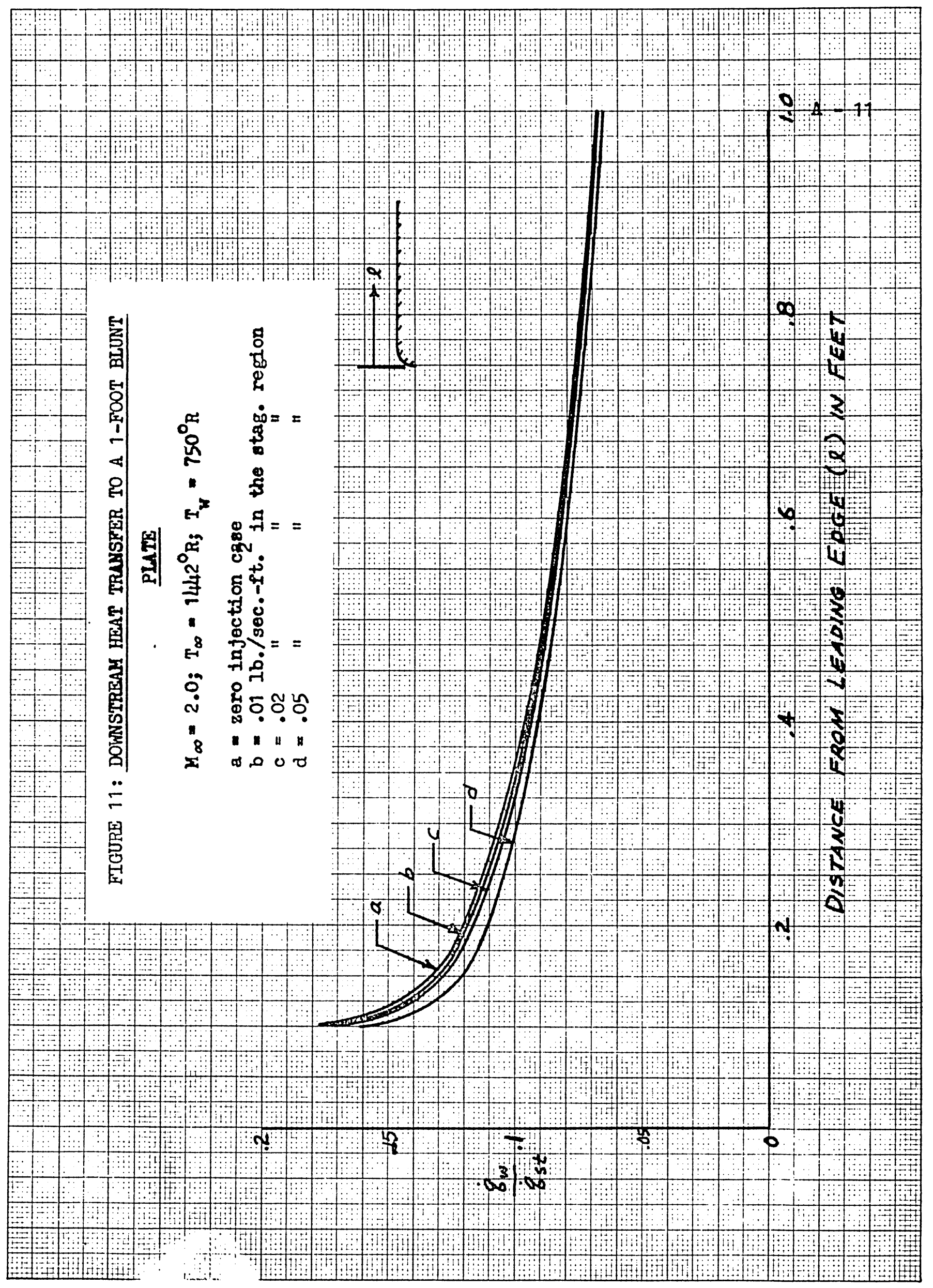




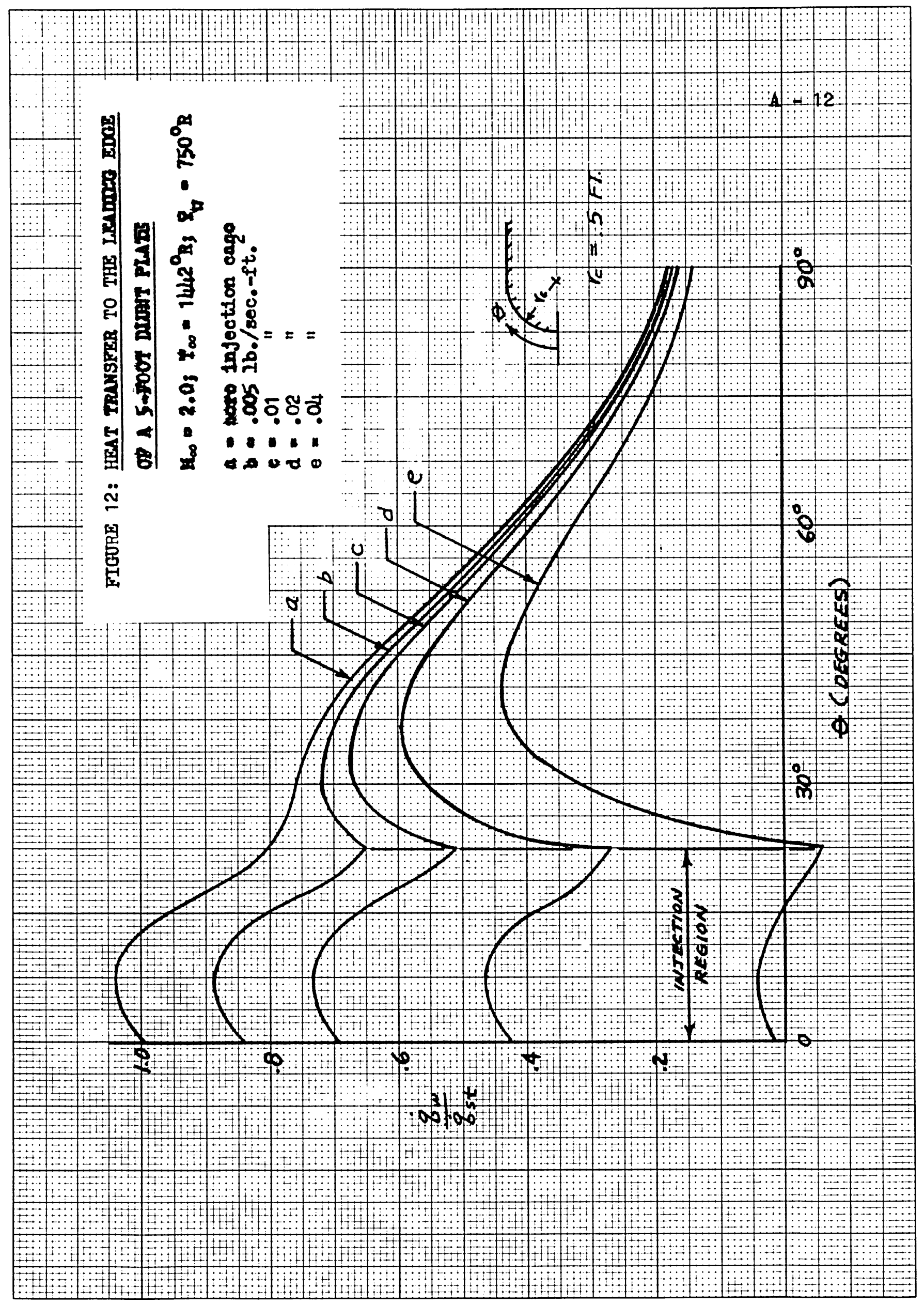




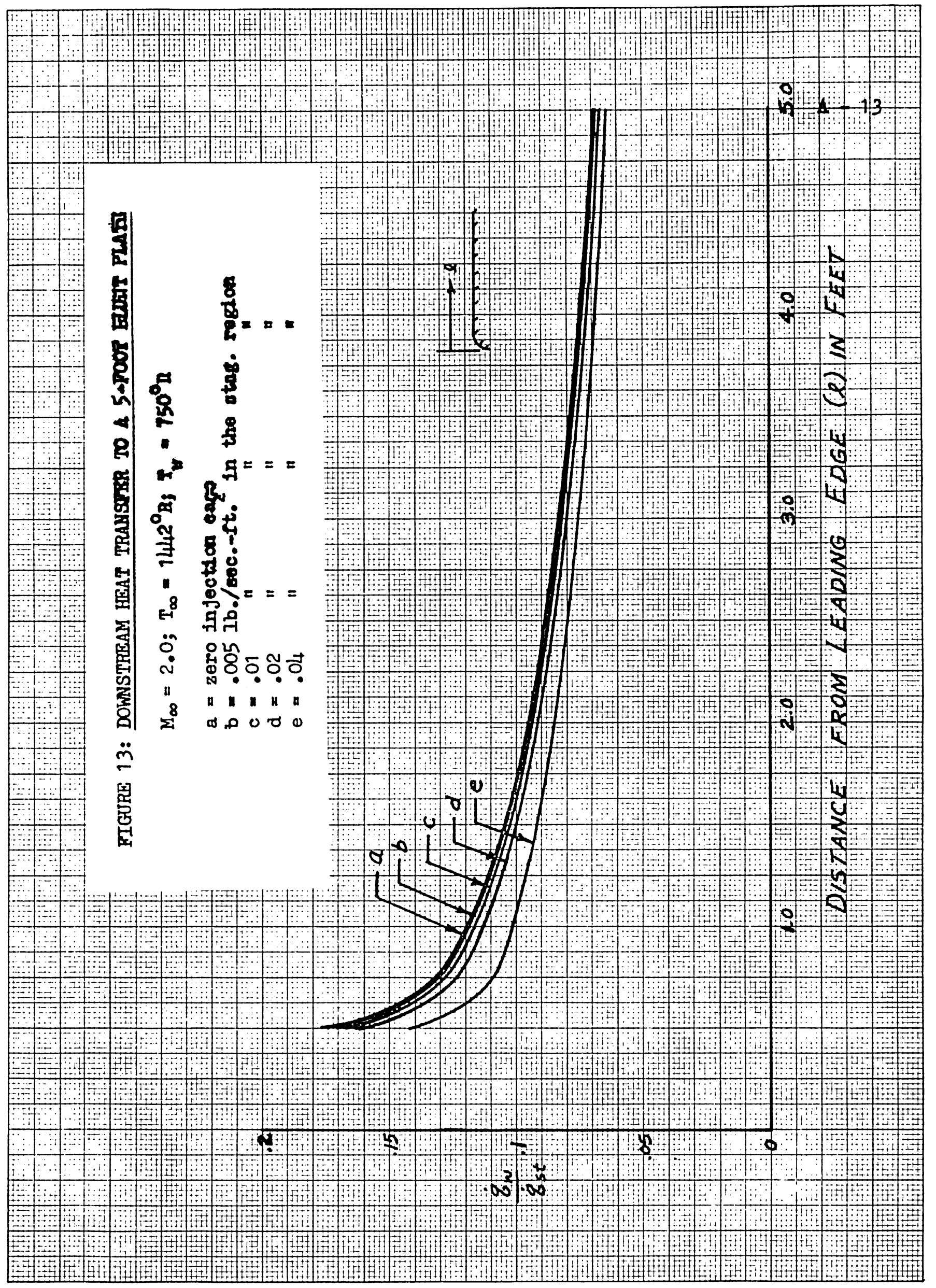




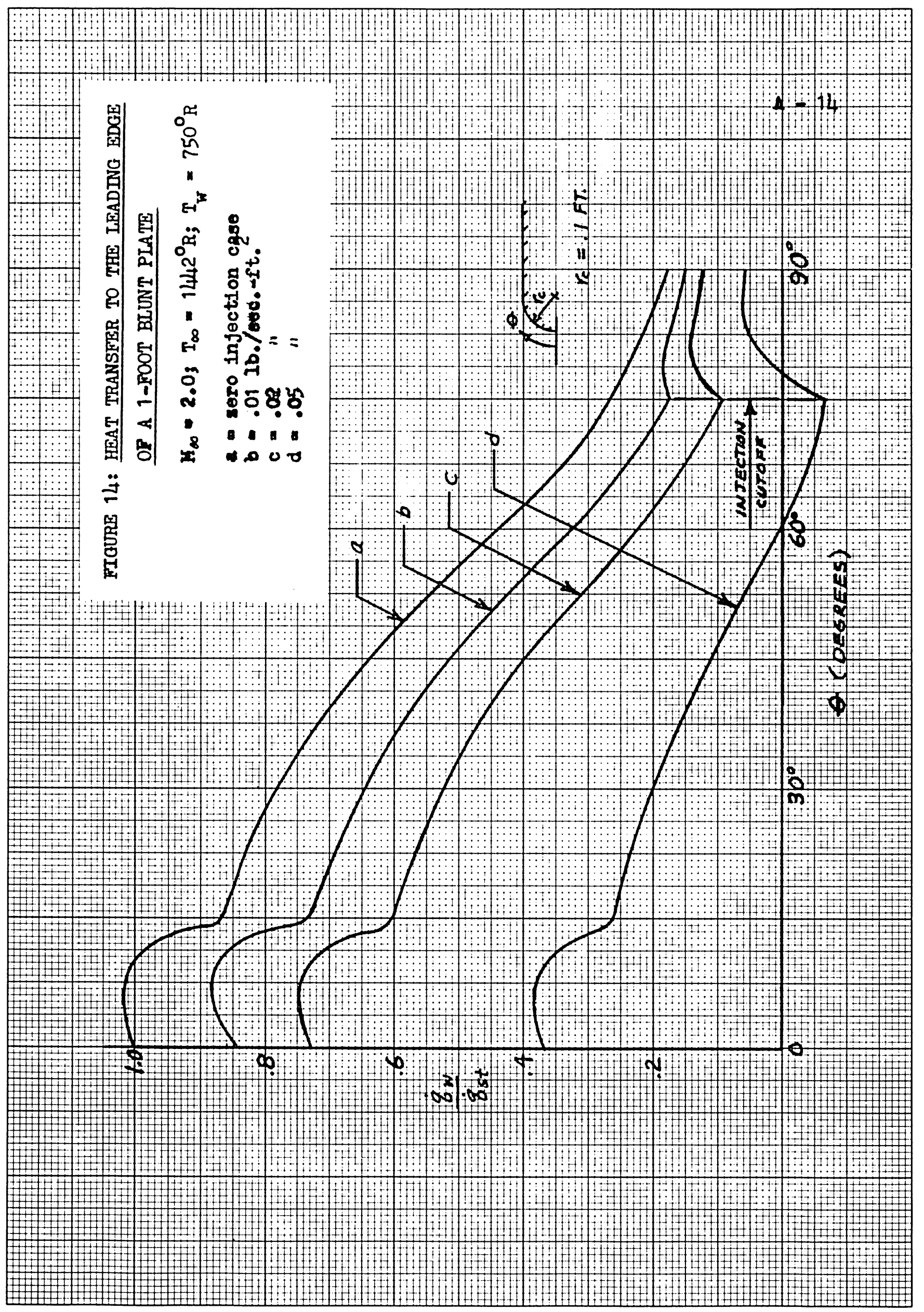




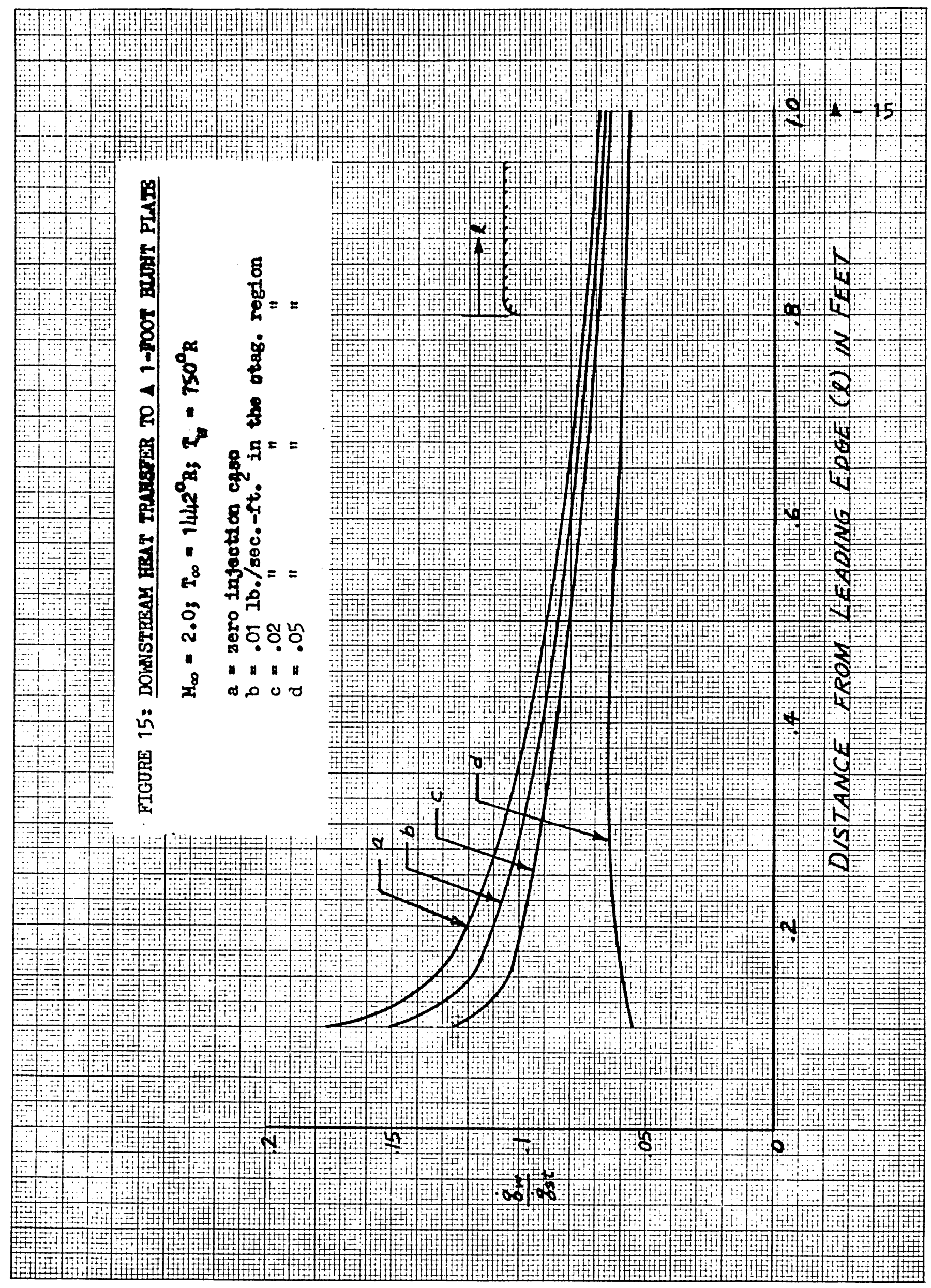




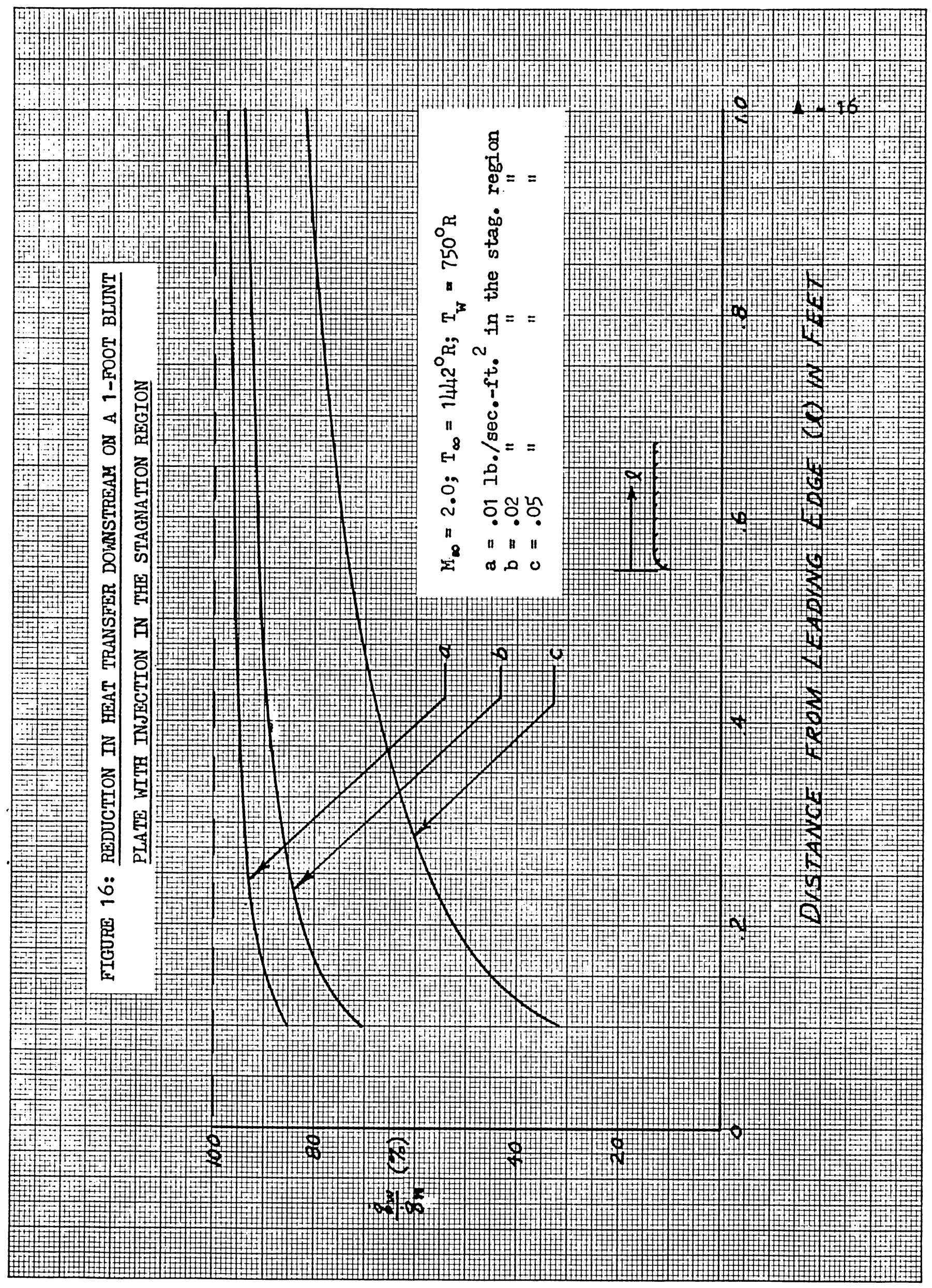

